CERN-PPE/92-198

17-November-1992

\title{
Analyzing the $e^{+} e^{-} \rightarrow e^{+} e^{-}$ angular distribution at LEP
}

\author{
P. Comas \\ Institut de Física d'Altes Energies \\ Universitat Autònoma de Barcelona, E-08193 Bellaterra (Barcelona), Spain \\ and \\ M. Martínez \\ CERN, CH-1211 Geneva, Switzerland
}

\begin{abstract}
The analysis of the lepton angular distribution at LEP is reviewed and the specific concerns in the case of $e^{+} e^{-}$final state are singled out. Studies of the physical information contained in the $e^{+} e^{-}$angular distribution are shown to justify the need for a new approach. Two different solutions that enable the complete use of the angular distribution information for the extraction of the $\mathrm{Z}$ parameters are presented. We show that these approaches have accuracies well above the future experimental needs.
\end{abstract}




\section{Introduction}

The present accuracy of LEP experiments in extracting physical information about electroweak parameters from measurements other than the lineshape one starts being considerable. For instance, the accuracy that can be obtained in determining the effective electroweak mixing angle from the combination of forward-backward and polarization asymmetries is, at present, equal to the one that can be obtained from the lineshape analysis [1]. In addition, the large statistics accumulated at the peak in the actual LEP running, will make the asymmetry measurements even more important.

Therefore, we must start considering the asymmetry measurements on the same foot as the lineshape ones on the requirement of accuracy on the experimental and on the theoretical side.

In this paper we shall concentrate on the discussion of the $e^{+} e^{-} \rightarrow e^{+} e^{-}$angular distribution. After the lineshape, the analysis of the lepton forward-backward asymmetry is at present the one giving the most accurate measurements of electroweak parameters at LEP [2]. The one in the process $e^{+} e^{-} \rightarrow e^{+} e^{-}$plays an important role due to the fact that this process is supposed to allow a direct study of the couplings of the $\mathrm{Z}$ to the electron, which have to be determined before extracting from the angular distributions the coupling of the $\mathrm{Z}$ to other fermions. In the angular distribution of the process $e^{+} e^{-} \rightarrow f \bar{f}$ there is no way of disentangling the information about $Z \rightarrow f \bar{f}$ without knowing beforehand the information about $Z \rightarrow e^{+} e^{-}$. This is possible since in the process $e^{+} e^{-} \rightarrow e^{+} e^{-}$the only couplings involved are those of the $Z$ to electrons.

In the framework of the Standard Model, careful studies of the implementation of the nonQED corrections have existed for some time [3] and the check of calculations performed by using different approaches has allowed a good understanding of the fact that the precision attained in this part of the problem is more than good enough [4]. At any rate, complete Standard Model semianalytical calculations for low and large angle Bhabha scattering have been available only since one year ago [5]. The main reason for this delay has been the problem of understanding the proper way of introducing the QED corrections with enough accuracy. Even now, these calculations are able to predict angular distributions just after a painful and expensive exercise of binning the angular region of interest and computing the integrated cross section in every bin. This panorama though, might change in the near future [6], [7].

Here we would like to present a rather different approach: a "model independent" parameterization useful for fitting purposes. The use of model independent formulae to describe the main measurements at the $Z$ resonance was suggested during the preparation for LEP-SLC physics [8]. When fitting to experimental data, a model independent formulation is a clean way of extracting the relevant physical information of the $Z$ resonance. The reasons for adopting a model independent formulation are twofold:

- Tests of the Standard Model may be biased if one assumes its validity from the beginning.

- By assuming only very basic theoretical concepts, one is able to analyze the results in terms of new theoretical models.

The basic idea of the model independent formulae is the fact that the $e^{+} e^{-}$interactions near the $Z$ pole, before considering pure photonic corrections, can be well described by Born-like diagrams in which effective couplings and propagators are used. The fermions are supposed to couple to the $Z$ with effective vector and axial couplings and the $Z$ propagator is described by a Breit-Wigner resonance, with s-dependent width. The photon exchange is described with 
couplings which are just the electric charge, and the usual QED dressed proton propagator is assumed. In this way, the well established QED ingredients are introduced in the calculation to allow disentangling the $Z$ phenomena.

As was the case for the $e^{+} e^{-} \rightarrow e^{+} e^{-}$lineshape until recent days [9], at present does not exist a completely satisfactory way of extracting the $Z$ parameters from the $e^{+} e^{-} \rightarrow e^{+} e^{-}$angular distribution.

The goal of this work is to analyze the $e^{+} e^{-} \rightarrow e^{+} e^{-}$angular distribution to justify the need for a new approach and present two different solutions based upon a parameterization in the same language used for the study of the s-driven processes. These approaches enable the full exploitation of the physical information contained in the $e^{+} e^{-} \rightarrow e^{+} e^{-}$angular distribution.

The outline of the paper is as follows. In section 2 we review the way physical information is extracted from the angular distribution in the case of s-driven processes to state, in the next section, the problems when trying to study the $e^{+} e^{-} \rightarrow e^{+} e^{-}$angular distribution. In section 3 we also analyze the physical information contained in the $e^{+} e^{-} \rightarrow e^{+} e^{-}$differential cross section near the $Z$ pole and compare different possible ways of trying to extract it concluding that two possible approaches are possible. In section 4 the DMIBA approach is presented. In section 5 we develop a fitting formula for the $e^{+} e^{-} \rightarrow e^{+} e^{-}$angular distribution in terms of physical parameters, show how they are connected to direct measurements of the Bhabha process and how the electroweak information can be extracted from the fit parameters. Section 6 deals with the inclusion of the missing QED corrections. Finally in section 7 we present the conclusions of our analysis.

\section{Analysis of the angular distribution in s-channel driven pro- cesses}

The information contained in the angular distribution of $e^{+} e^{-} \rightarrow \mu^{+} \mu^{-}$is extracted in two steps:

- first the forward-backward asymmetry is extracted from the data, by computing directly

$$
A_{F B}=\frac{\sigma_{F}-\sigma_{B}}{\sigma_{F}+\sigma_{B}}
$$

or by fitting the angular distribution with a simple formula

$$
\frac{d \sigma}{d \cos \theta}=C\left(1+\cos ^{2} \theta+\frac{8}{3} A_{F B} \cos \theta\right)
$$

where, $\theta$ is taken as the polar angle in the reduced center-of-mass frame ${ }^{1}$. The first definition has the advantage of being completely general and, hence, applicable to any process. The second definition, assumes a given behaviour of the angular distribution but, under this assumption, allows a more accurate determination of $A_{F B}$ since the whole angular distribution is used instead of just two angular bins (as is the case in the first method). This results in an increase of accuracy of the order of $5 \%$. In addition, in the second procedure, if a log-likelihood method is applied to fit the data, then there is no need of knowing at all the angular response of the apparatus (provided it is charge-symmetric or forward-backward symmetric).

\footnotetext{
${ }^{1}$ Some experiments use also this formula taking $\theta$ as the polar angle in the lab frame. It has been shown that, in practice, up to a good degree of accuracy, the choice of a given definition of the scattering angle is just matter of taste and convenience [10].
} 
- second, the data for $A_{F B}(s)$ is fitted to a "model independent" formula in which photonic corrections are also explicitly incorporated. This allows disentangling the pure photonic effects (the ones due to radiation as well as the ones due to the $\gamma$ exchange contribution) from the rest. It enables also taking properly into account the energy dependence of the asymmetry to add-up the information from all the energy points. The fit is done simultaneously with the lineshape data or alternatively, by incorporating as constraints the information obtained from the lineshape analysis. In this way, information about $v$ and $a$ is obtained. These couplings can be interpreted as absorbing all sort of corrections not explicitly accounted for in the fitting formulae, namely real parts of $Z$ vacuum polarization, $\gamma-Z$ mixing, weak vertices and other small effects like weak boxes ${ }^{2}$.

In this procedure, regardless on the way used to compute $A_{F B}$ from the data, the basic assumption is that all the information of the angular distribution is just the one in $A_{F B}$. We shall see now that, indeed, this is a very good approximation for the s-channel driven processes, but not necessarily for $e^{+} e^{-} \rightarrow e^{+} e^{-}$.

The reason for this assumption being very precise for s-driven processes is exactly the same as the one allowing the use of equation (2) to obtain it from the data: the fact that a formula with just one free parameter (besides the global normalization which can be identified as the total cross section measurement) is enough to describe the angular distribution. This is due to the particular structure of the angular distribution in the s-channel. At the Born level, the angular distribution is well known to be

$$
\begin{aligned}
\frac{d \sigma^{0}}{d \cos \theta} & =\left(\frac{d \sigma^{0}}{d \cos \theta}\right)_{Z_{s} Z_{s}}+\left(\frac{d \sigma^{0}}{d \cos \theta}\right)_{Z_{s} \gamma_{s}}+\left(\frac{d \sigma^{0}}{d \cos \theta}\right)_{\gamma_{s} \gamma_{s}} \\
& =\frac{\pi \alpha^{2}}{2 s} \frac{s^{2}}{|Z(s)|^{2}}\left[\left(1+\cos ^{2} \theta\right)\left(v^{2}+a^{2}\right)^{2}+8 \cos \theta v^{2} a^{2}\right] \\
& +\frac{\pi \alpha^{2}}{2 s} \frac{s\left(s-M_{Z}^{2}\right)}{|Z(s)|^{2}}\left[\left(1+\cos ^{2} \theta\right) 2 v^{2}+\cos \theta 4 a^{2}\right] \\
& +\frac{\pi \alpha^{2}}{2 s}\left(1+\cos ^{2} \theta\right) \\
|Z(s)|^{2} & =\left(s-M_{Z}^{2}\right)^{2}+\left(M_{Z} \Gamma_{Z}\right)^{2}
\end{aligned}
$$

so that it can be written as

$$
\frac{d \sigma^{0}}{d \cos \theta}=C^{0}(s)\left(1+\cos ^{2} \theta+\frac{8}{3} A_{F B}^{0}(s) \cos \theta\right)
$$

being

$$
C^{0}(s)=\frac{3}{8} \sigma^{0}(s)=\frac{\pi \alpha^{2}}{2 s}\left[\frac{s^{2}}{|Z(s)|^{2}}\left(v^{2}+a^{2}\right)^{2}+\frac{s\left(s-M_{Z}^{2}\right)}{|Z(s)|^{2}} 2 v^{2}+1\right]
$$

and

$$
\begin{aligned}
A_{F B}^{0}(s) & =\frac{\sigma_{F}^{0}(s)-\sigma_{B}^{0}(s)}{\sigma^{0}(s)} \\
& =\frac{1}{\sigma^{0}(s)} \frac{\pi \alpha^{2}}{2 s}\left[\frac{s^{2}}{|Z(s)|^{2}} 8 v^{2} a^{2}+\frac{s\left(s-M_{Z}^{2}\right)}{|Z(s)|^{2}} 4 a^{2}\right]
\end{aligned}
$$

\footnotetext{
${ }^{2}$ The imaginary part of the $\gamma$ vacuum polarization, which plays a relevant role in $A_{F B}$ is included explicitly in the fitting formulae. This is so since, like for the real part, its value can be precisely predicted in QED and therefore can be considered within in the photonic corrections.
} 
The picture does not change at all when non-photonic corrections are applied, since they can be implemented by doing just some simple replacements, namely:

$$
\begin{aligned}
& \begin{aligned}
\alpha \rightarrow \alpha(s) & \text { Real part of } \gamma \text { self-energy } \\
s\left(s-M_{Z}^{2}\right) \rightarrow s\left(s-M_{Z}^{2}\right)+s^{2} \frac{\Gamma_{Z}}{M_{Z}} \operatorname{Im}(\Delta \alpha) & \text { Imaginary part of } \gamma \text { self-energy }
\end{aligned} \\
& |Z(s)|^{2} \rightarrow\left(s-M_{Z}^{2}\right)^{2}+\left(s \frac{\Gamma_{Z}}{M_{Z}}\right)^{2} \quad \text { Imaginary part of } Z \text { self energy } \\
& v, a \rightarrow \sqrt{F_{G}(s)} g_{V}, \sqrt{F_{G}(s)} g_{A} \quad \text { Real part of } Z \text { self energy, } \gamma-Z \text { mixing, } \\
& \text { weak vertices }
\end{aligned}
$$

where

$$
F_{G}(s)=\frac{G_{F} M_{Z}^{2}}{2 \sqrt{2} \pi \alpha(s)}
$$

These replacements transform $C^{0} \rightarrow \hat{C}$ and $A_{F B}^{0} \rightarrow \hat{A}_{F B}$. Concerning photonic corrections, since the dominant ones are initial state radiation, it is rather easy to show that they do not affect sizably the simple angular distribution described in equation (2). Let's take just initial state radiation in the collinear limit and let's include it by convoluting the reduced differential cross section with the probability distributions describing the photonic radiation $H(z, s)$, where $1-z$ denotes the fractional energy carried out by photons:

$$
\begin{aligned}
\frac{d \sigma}{d \cos \theta}(s) & =\int_{0}^{1} H(z, s)\left[\hat{C}(z s)\left(1+\cos ^{2} \theta+\frac{8}{3} \hat{A}_{F B}(z s) \cos \theta\right)\right] d z \\
& =C(s)\left(1+\cos ^{2} \theta+\frac{8}{3} A_{F B}(s) \cos \theta\right)
\end{aligned}
$$

being then

$$
\begin{aligned}
C(s) & =\int_{0}^{1} H(z, s) \hat{C}(z s) d z=\frac{3}{8} \sigma(s) \\
A_{F B}(s) & =\frac{1}{C(s)} \int_{0}^{1} H(z, s) \hat{C}(z s) \hat{A}_{F B}(z s) d z
\end{aligned}
$$

Therefore, the experimental analysis procedure is very clear: the forward-backward asymmetry is obtained for every energy by either one of the methods discussed and afterwards it is fitted with the radiative equation (11).

\section{Physics from the $e^{+} e^{-} \rightarrow e^{+} e^{-}$angular distribution}

In the case of $e^{+} e^{-} \rightarrow e^{+} e^{-}$, the Born cross section has ten different terms,

$$
\begin{aligned}
\left(\frac{d \sigma^{0}}{d \cos \theta}\right)_{Z_{s} Z_{s}} & \left.=\frac{\pi \alpha^{2}}{2 s} \frac{s^{2}}{\left(s-M_{Z}^{2}\right)^{2}+\left(M_{Z} \Gamma_{Z}\right)^{2}}\left[\left(1+\cos ^{2} \theta\right)\left(v^{2}+a^{2}\right)^{2}+8 \cos \theta v^{2} a^{2}\right)\right] \\
\left(\frac{d \sigma^{0}}{d \cos \theta}\right)_{\gamma_{t} \gamma_{t}} & =\frac{\pi \alpha^{2}}{2 s} 2 \frac{4+(1+\cos \theta)^{2}}{(\cos \theta-1)^{2}} \\
\left(\frac{d \sigma^{0}}{d \cos \theta}\right)_{Z_{s} \gamma_{t}} & =\frac{\pi \alpha^{2}}{2 s} \frac{s\left(s-M_{Z}^{2}\right)}{\left(s-M_{Z}^{2}\right)^{2}+\left(M_{Z} \Gamma_{Z}\right)^{2}} 2\left(v^{2}+a^{2}\right) \frac{(1+\cos \theta)^{2}}{(\cos \theta-1)}
\end{aligned}
$$




$$
\begin{aligned}
& \left(\frac{d \sigma^{0}}{d \cos \theta}\right)_{\gamma_{s} \gamma_{s}}=\frac{\pi \alpha^{2}}{2 s}\left(1+\cos ^{2} \theta\right) \\
& \left(\frac{d \sigma^{0}}{d \cos \theta}\right)_{\gamma_{s} \gamma_{t}}=\frac{\pi \alpha^{2}}{2 s} 2 \frac{(1+\cos \theta)^{2}}{(\cos \theta-1)} \\
& \left(\frac{d \sigma^{0}}{d \cos \theta}\right)_{Z_{s} \gamma_{s}}=\frac{\pi \alpha^{2}}{2 s} \frac{s\left(s-M_{Z}^{2}\right)}{\left(s-M_{Z}^{2}\right)^{2}+\left(M_{Z} \Gamma_{Z}\right)^{2}}\left[\left(1+\cos ^{2} \theta\right) 2 v^{2}+\cos \theta 4 a^{2}\right] \\
& \left(\frac{d \sigma^{0}}{d \cos \theta}\right)_{Z_{t} Z_{t}}=\frac{\pi \alpha^{2}}{2 s} \frac{s^{2}}{\left(t-M_{Z}^{2}\right)^{2}} \frac{1}{2}\left[(1+\cos \theta)^{2}\left(\left(v^{2}+a^{2}\right)^{2}+4 v^{2} a^{2}\right)+4\left(\left(v^{2}+a^{2}\right)^{2}-4 v^{2} a^{2}\right)\right] \\
& \left(\frac{d \sigma^{0}}{d \cos \theta}\right)_{Z_{s} Z_{t}}=\frac{\pi \alpha^{2}}{2 s} \frac{s\left(s-M_{Z}^{2}\right)}{\left(s-M_{Z}^{2}\right)^{2}+\left(M_{Z} \Gamma_{Z}\right)^{2}} \frac{s}{t-M_{Z}^{2}}(1+\cos \theta)^{2}\left(\left(v^{2}+a^{2}\right)^{2}+4 v^{2} a^{2}\right) \\
& \left(\frac{d \sigma^{0}}{d \cos \theta}\right)_{\gamma_{t} Z_{t}}=\frac{\pi \alpha^{2}}{2 s} \frac{s}{t-M_{Z}^{2}} \frac{2}{\cos \theta-1}\left[(1+\cos \theta)^{2}\left(v^{2}+a^{2}\right)+4\left(v^{2}-a^{2}\right)\right] \\
& \left(\frac{d \sigma^{0}}{d \cos \theta}\right)_{\gamma_{s} Z_{t}}=\frac{\pi \alpha^{2}}{2 s} \frac{s}{t-M_{Z}^{2}}(1+\cos \theta)^{2}\left(v^{2}+a^{2}\right)
\end{aligned}
$$

Therefore, it is easy to understand that some of the nice features of the cross section for s-driven processes are not present here, namely:

- first it is clear that, even in the Born case, there are more parameters needed to describe completely the angular distribution, since the dependence on $\cos \theta$ is much richer and complicated. Therefore, the analysis in two steps with just an intermediate parameter $A_{F B}$ collapsing the information of the angular distribution is, in principle, not justified.

- second, it is not obvious that the inclusion of non-QED corrections will be possible like in the previous case, preserving the number of tree level parameters and giving them the meaning of effective parameters. Clearly, there are two different scales in the problem that now will show up when considering loop corrections: $s$ and t. Therefore, it seems reasonable considering a set of effective couplings at the scale $s$ and another one at the scale $t$ absorbing the corrections in the respective diagrams.

- third, given the fact that detection cuts in this case are mandatory to keep the cross section finite, it is not evident that QED corrections can be properly included by a simple convolution like the one given by equation (10). Therefore a different treatment has to be applied [5].

\subsection{Sensitivity function for the differential Bhabha cross section}

At any rate, we can use equations (12) to study in a semi-quantitative way which is actually the physics contained in the angular distribution for $e^{+} e^{-} \rightarrow e^{+} e^{-}$. In order to do that, following the ideas of [9], we will use the language of sensitivity functions with the particularities explained in the appendix.

To perform this analysis, we will forget about the fact that the $e^{+} e^{-} \rightarrow e^{+} e^{-}$differential cross section in the language of effective couplings is a function of four electroweak couplings $(v(s), a(s), v(t)$ and $a(t))$ instead of just two and will take $v=v(s)$ and $a=a(s)$, freezing $v(t)$ and $a(t)$ to their Standard Model values ${ }^{3}$.

\footnotetext{
${ }^{3}$ This is justified by the fact that the t-channel couplings $v(t)$ and $a(t)$ are present just in the contributions
} 
To understand which new information can be extracted from the angular distribution with respect to the one already present in the lineshape, we are now ready to change variables to $\Gamma_{e}$, basically determined from the lineshape analysis in the case of the s-channel processes, and $A_{F B}^{0}$, the new measurement which comes from the asymmetry, using the tree level relations

$$
\begin{aligned}
\Gamma_{e} & \equiv \frac{\alpha M_{Z}}{3}\left(v^{2}+a^{2}\right) \\
A_{F B}^{0} & \equiv \frac{3}{4} \cdot\left(\frac{2 v a}{v^{2}+a^{2}}\right)^{2}
\end{aligned}
$$

As in [9], we will compare three different cases:

- when the complete matrix element squared is used,

- when only the s-channel diagrams are taken into account. This approach will be used as a reference to understand the differences when considering also t-channel exchange, and

- when the complete matrix element squared is used, but all the non-s-channel contributions are frozen ("t-channel subtraction or addition").

For that, three different approaches with increasing complexity have been followed (reflecting different ways used so far in the literature to attack the problem):

(1) Use only the $A_{F B}$ measurement.

We study the sensitivity of just the forward-backward asymmetry with respect to $A_{F B}^{0}$ asumming that the information from $\Gamma_{e}$ is fixed by the lineshape analysis (see the appendix for details on the definition of the sensitivity function).

As we are freezing completely the non-s-channel contribution, there is nice agreement for all energies between the complete approach and the "t-channel addition" one (figures 2). This is not realistic as the sensitivity of the forward-backward asymmetry to $A_{F B}^{0}$ will be affected by the actual uncertainty in $\Gamma_{e}$. Therefore, since in the case of $e^{+} e^{-} \rightarrow e^{+} e^{-}$clearly the interpretation in terms of parameters of the lineshape measurement and the forward-backward asymmetry measurement are intrinsically connected, we must follow a different approach,

(2) Use both, the $\sigma$ and the $A_{F B}$ measurements simultaneously.

We study the combined sensitivity of the cross section and the forward-backward asymmetry, with respect to either one of the parameters we want to measure $\Gamma_{e}$ or $A_{F B}^{0}$. To do so, we need a different definition of the sensitivity function which is discussed in detail in the appendix.

In the study of the combined sensitivity with respect to $\Gamma_{e}$, (figure 3 ) we basically reproduce the results of ref. [9]. This means that the forward-backward asymmetry does not enrich the measurement of $\Gamma_{e}$ and therefore, that all the assumptions therein were correct.

Concerning the combined sensitivity with respect to $A_{F B}^{0}$ the picture changes remarkably. From figures 4 we see that, at the peak, where the interference terms vanish, there is perfect agreement between the complete case and the "t-channel addition method", as it should be, but that this agreement disappears when moving from the peak. The reason is that as we move from the peak, the interference terms become more important, and then $\Gamma_{e}$ starts playing a role. Like in the case of the sensitivity with respect to $\Gamma_{e}$, below the peak, the "t-channel addition"

which come from the t-channel $Z$ exchange, which are a rather small correction to the differential cross section (see figures 1) and the sensitivity to this couplings is very small. 
underestimates the real sensitivity (due to the fact that the sensitivity of the interference is positive below the peak) whereas above the peak, the effect is roughly the opposite. In any case, the size of the differences is well below the one for $\Gamma_{e}$ : a difference of about $20 \%$ which for $\Gamma_{e}$ shows up already at $1 \mathrm{GeV}$ from the peak, for $A_{F B}^{0}$ shows up just for points further than $3 \mathrm{GeV}$ from the peak.

As in [9], for the complete and "t-channel addition" cases, maximal sensitivity is reached at a specific value of $\cos \theta$ depending on $\sqrt{s}$. Also the fact that the Bhabha process sensitivity is smaller than in pure $Z$ processes is clear from the figures.

Finally, figure $4 \mathrm{~d}$ shows the three sensitivities as a function of $\sqrt{s}$ for an angular range of $|\cos \theta|<0.9$. This bias in the sensitivity is very important on the wings. Although, if the luminosity is distributed symmetrically with respect to the $Z$ peak, there is an approximate cancellation of the underestimation and overestimation, this cancellation is accidental so it would be better to use an approach in which the complete Bhabha angular distribution is fitted to the data.

In any case, as we said before, the rich $\cos \theta$ dependence of the $e^{+} e^{-} \rightarrow e^{+} e^{-}$angular distribution, suggests that we might lose information by just using the integrated cross section and the forward-backward asymmetry as observables. To check this point and allow for the most general analysis of the differential cross section, we undertake a last approach,

\section{(3) Use the complete angular distribution measurement.}

We study the sensitivity of the whole angular distribution (normalization included) with respect to either one of the parameters we want to measure $\Gamma_{e}$ or $A_{F B}^{0}$. The definition and properties of the integrated sensitivity function are discussed in the appendix.

By comparing the sensitivities obtained this way (figures 5) with the ones obtained in the previous approach, we see that now the sensitivity is slightly larger and that, for the complete and "t-channel addition" cases, it doesn't decrease after a certain $\cos \theta$ value but remains flat ${ }^{4}$. The reason it the following: although increasing the acceptance means, besides adding more events from the annihilation diagram, enlarging the contribution from the sensitivity-degrading photon-t-channel diagram as in the combined approach, now the thorough exploitation of our knowledge of the angular distribution lets us know precisely the contribution of each diagram when we increase the acceptance and thus, neither enlarge nor reduce the sensitivity when the contribution of the photon-t-channel is dominant.

Therefore, apart from the numerical importance of not freezing the "t-channel" contribution that can be seen in the figures presented, the main conclusion of the sensitivity studies is that there is additional information in the Bhabha differential cross section which cannot simply be reduced to the one in the integrated cross section and forward-backward asymmetry measurements.

Two overcome this problem we have set up two different approaches:

- an analysis of the complete angular distribution in one step in which the event angular distribution is directly fitted to a "model independent" formula in terms of the $Z$ parameters using, for instance, a log-likelihood maximization, and thus, avoiding the drawbacks of the "t-channel subtraction or addition" methods,

\footnotetext{
${ }^{4}$ We have checked that if the angular distribution is just simplified to two bins of information, then the integrated sensitivity is precisely equal to the combined sensitivity of lineshape and forward-backward asymmetries.
} 
- an analysis of the complete angular distribution in two steps, like for the s-channel processes. In the first step the whole angular distribution information is collapsed into a number of quantities (more than two) by fitting it to a simple formula containing the different terms which add up in the observed angular dependence. This quantities are chosen such that they can also be computed directly from the observed angular distribution without any further assumption (therefore, without any fit). In the second step, these quantities are fitted, together with other measurements in a "model independent fit" to obtain the electroweak parameters.

The second approach seems to us more convenient because it matches better the present experimental procedure applied by LEP experiments and enables the quotation of just few quantities instead of the full angular distributions. However, for completeness, both approaches will be presented next.

\section{Direct fit to the angular distribution: DMIBA approach}

MIBA [9] is a fast computer program providing a "model independent" description of the integrated $e^{+} e^{-}$cross section which incorporates leading $\log$ QED corrections up to $O\left(\alpha^{2}\right)$ and soft photon resumation following the ALIBABA scheme.

Given the fact that there we use a center-of-mass definition of the scattering angle which is a natural integration variable for the differential cross section, nothing prevents us from going a step ahead and changing the order in which the different integrations are done to have as the external most the one over $\cos \theta$. Attention must be paid to the proper treatment of the integration limits in changing the integration order. Anyway, since the integration is performed numerically, this can be implemented in practice with a simple Heaviside function. Once this is done, we can just eliminate this integration and we end up with a calculation able to predict directly the differential cross section:

$$
\frac{d \sigma}{d \cos \theta}(s, \cos \theta)=\int_{0}^{1} d x_{+} \int_{0}^{1} d x_{-} \Theta\left(x_{+}, x_{-}, \cos \theta\right) D\left(x_{+}, s\right) D\left(x_{-}, s\right) \frac{d \hat{\sigma}\left(s x_{+} x_{-}, \cos \theta\right)}{d \cos \theta}
$$

being $D(x, s)$ the electron structure function and $\Theta\left(x_{+}, x_{-}, \cos \theta\right)$ a Heaviside function implementing the kinematical limits and the cut conditions. This is what we will call the DMIBA approach.

Since all the needed steps are just mathematical manipulations, the physical accuracy of the DMIBA approach is as good as the one of MIBA.

The actual implementation of the QED corrections in DMIBA follows the scheme of ref. [5]. At any rate, this scheme can be greatly simplified if a rapidity cut $Y_{\text {cut }}$ (see section 5.2) is applied. From the calculational point of view, the use of a rapidity cut has the advantage that the radiator function $H\left(z, Y_{c u t}, s\right)$ can be computed analyticaly up to $O\left(\alpha^{2}\right)$ [11] and therefore we are left with just one integration:

$$
\frac{d \sigma}{d \cos \theta}(s, \cos \theta)=\int_{0}^{1} d z H\left(z, Y_{c u t}, s\right) \frac{d \hat{\sigma}(z s, \cos \theta)}{d \cos \theta}
$$

instead of the two-dimensional integration needed when an acollinearity cut is used [5]. This results in the actual computational time being much shorter and the calculational results being more robust. 
Figure 6 shows the agreement between the ALIBABA prediction and the DMIBA function for the binned angular distribution.

\section{Development of a fitting formula for the $e^{+} e^{-} \rightarrow e^{+} e^{-}$angular distribution}

At any rate, inspired in the s-channel processes, we can also write the Born angular distribution in a way where the physical information is more apparent:

$$
\begin{aligned}
\frac{d \sigma^{0}}{d \cos \theta} & =C^{0}(s)\left(1+\cos ^{2} \theta+\frac{8}{3} A_{F B}^{0}(s) \cos \theta\right) \\
& +A_{\text {int }}^{0}(s, t) \frac{(1+\cos \theta)^{2}}{(\cos \theta-1)}+A_{\gamma t}^{0}(s, t) \frac{4+(1+\cos \theta)^{2}}{(\cos \theta-1)^{2}} \\
& +\left(\frac{d \sigma^{0}}{d \cos \theta}\right)_{Z_{t} Z_{t}}+\left(\frac{d \sigma^{0}}{d \cos \theta}\right)_{Z_{s} Z_{t}}+\left(\frac{d \sigma^{0}}{d \cos \theta}\right)_{\gamma_{t} Z_{t}}+\left(\frac{d \sigma^{0}}{d \cos \theta}\right)_{\gamma_{s} Z_{t}}
\end{aligned}
$$

being $C^{0}(s)$ and $A_{F B}^{0}(s)$ the same with the same meaning as in the s-channel processes and

$$
A_{\text {int }}^{0}(s, t)=\frac{\pi \alpha^{2}}{s}\left[\frac{s\left(s-M_{Z}^{2}\right)}{|Z(s)|^{2}}\left(v^{2}+a^{2}\right)+1\right]
$$

and

$$
A_{\gamma t}^{0}(s, t)=\frac{\pi \alpha^{2}}{s}
$$

Clearly, $A_{i n t}^{0}(s, t)$ and $A_{\gamma t}^{0}(s, t)$ are new parameters which give us some information that does not appear in the s-channel driven processes. $A_{\text {int }}^{0}(s, t)$ accounts for the information coming from $Z_{s} \gamma_{t}$ and $\gamma_{s} \gamma_{t}$ interference terms. $A_{\gamma t}^{0}(s, t)$ is a pure QED prediction and, therefore, its actual determination from the data would provide us an independent way of measuring the luminosity with large angle Bhabha.

Finally, the contribution of the last four $Z_{t}$ terms is very small and, therefore, can be treated as a correction to the other dominant part.

At this point, as in s-channel processes, we have a "model independent" formula with four parameters which describes our Bhabha Born angular distribution.

\subsection{Inclusion of non-photonic corrections}

For the dominant part, non-photonic corrections can be implemented by doing just simple replacements, as for s-channel driven processes. Besides the ones we had there (8), the coupling constant $\alpha(t)$ will be changed as follows,

$$
\alpha(t) \rightarrow \alpha(s) \frac{\alpha\left(-\frac{1}{2} M_{Z}^{2}(1-\cos \theta)\right)}{\alpha\left(M_{Z}^{2}\right)}
$$

in order to decouple the angular dependence from the energy dependence in the running of the electromagnetic coupling constant in the t channel. This splitting is justified by the clear dominance of the $M_{Z}$ scale. 
For the other four $Z_{t}$ terms we will keep the expressions as in (12) after changing the $Z$ propagator as before and evaluating the coupling constants corresponding to the $Z_{t}$ diagrams at a scale $\bar{t}$. The scale $\bar{t}$ is somewhat arbitrary (as final results do not depend on the precise choice of $\bar{t}$ ) and we have chosen it as the mean value of $t$ in the integration range. Finally, the couplings $v(t)$ and $a(t)$ we have here are fixed to their Standard Model values. This, as we said, can be done because the contribution of these terms is very small and the assumption on the model only enters as subleading effect.

\subsection{Inclusion of photonic corrections}

When considering the effect of radiation, it becomes obvious that it would be highly desirable the use of a variable allowing to limit the amount of radiated energy without changing the $\cos \theta$ dependence of the kernel process. If such variable can experimentally be used, then the simple dressed-Born angular distribution will be maintained. This, as pointed out in [12], is accomplished by a cut in rapidity, defined as

$$
Y=\frac{1}{2} \ln \frac{x_{+}}{x_{-}}
$$

where $x_{+}$and $x_{-}$are the fractional energies left to the positron and the electron respectively after radiation. This definition, in the collinear radiation limit is connected to the final state angles directly measured experimentally:

$$
Y=\ln \sqrt{\frac{\left.\sin \theta_{+}\left(1+\cos \theta_{-}\right)+\sin \theta_{-}\left(1+\cos \theta_{+}\right)\right)}{\left.\sin \theta_{+}\left(1-\cos \theta_{-}\right)+\sin \theta_{-}\left(1-\cos \theta_{+}\right)\right)}}
$$

where $\theta_{+}\left(\theta_{-}\right)$is the polar angle of the observed positron (electron) in the lab frame. Since this kinematical configuration constitutes, by far, the bulk of the radiation effects, this last equation can be considered as the practical definition of the rapidity for experimental purposes (even in the cases in which non-collinear radiation is observed). As we will see in section 6, the identification of the two previous definitions will require applying small corrections at some point.

A rapidity cut acts, in fact, as an angle-dependent acollinearity cut, as can be seen in figure 7 . In that figure we can see that, in practice, the change from an acollinearity cut to a rapidity cut is just sizable in processes where hard radiation is more likely and with a peaked differential cross section at low angles, just as Bhabha scattering is, due to the contribution of the $\gamma_{t}$ exchange diagram.

\subsection{Physical fitting formula}

Once all non-photonic as well as photonic corrections have been included, we get a "model independent" fitting formula with four physical parameters which lets us fit the Bhabha radiative differential cross section in terms of the Born angular dependence, just as in the s-channel case,

$$
\begin{aligned}
\frac{d \sigma}{d \cos \theta} & =C(s)\left(1+\cos ^{2} \theta+\frac{8}{3} A_{F B}(s) \cos \theta\right) \\
& +A_{\text {int }}(s) \frac{\alpha\left(-\frac{1}{2} M_{Z}^{2}(1-\cos \theta)\right)}{\alpha\left(M_{Z}^{2}\right)} \frac{(1+\cos \theta)^{2}}{(\cos \theta-1)}
\end{aligned}
$$




$$
\begin{aligned}
& +A_{\gamma t}(s) \frac{\alpha^{2}\left(-\frac{1}{2} M_{Z}^{2}(1-\cos \theta)\right)}{\alpha^{2}\left(M_{Z}^{2}\right)} \frac{4+(1+\cos \theta)^{2}}{(\cos \theta-1)^{2}} \\
& +X_{1}(s)\left(\frac{d \hat{\sigma}}{d \cos \theta}\right)_{Z_{t} Z_{t}}+X_{2}(s)\left(\frac{d \hat{\sigma}}{d \cos \theta}\right)_{Z_{s} Z_{t}} \\
& +\quad X_{3}(s)\left(\frac{d \hat{\sigma}}{d \cos \theta}\right)_{\gamma_{t} Z_{t}}+X_{4}(s)\left(\frac{d \hat{\sigma}}{d \cos \theta}\right)_{\gamma_{s} Z_{t}}
\end{aligned}
$$

where now, all the corrections are taken into account in the four parameters $C(s), A_{F B}(s)$, $A_{\text {int }}(s), A_{\gamma t}(s)$.

The $X_{i}(s)$ are small energy dependent coefficients needed to dress the $Z_{t}$ terms and give the desired degree of precision for the fitting formula. Their precise value is obtained as the ratio between the corresponding photonic corrected $Z_{t}$ term calculated using DMIBA to the non-photonic corrected one, both at $\cos \theta=0$.

At this point, we could also make use of our knowledge of the luminosity (obviously much better determined from low angle Bhabha scattering), and hence, of $A_{\gamma t}(s)$, to improve the errors on the other three parameters.

\subsection{Connection with direct measurements}

The four parameters introduced in the previous section have two attractive features:

- on the one hand, they constitute a superset of the parameters used in the description of the s-channel processes to which everyone is familiar and hence allows a simple understanding of which is the additional information contained in the $e^{+} e^{-}$angular distribution and,

- on the other hand, every parameter is connected with a single piece of the theoretical angular distribution and has a simple interpretation in terms of the diagrammatics describing the process at tree level.

Unfortunately they have also two important drawbacks:

- Unlike in the case of the s-channel processes, these parameters do not correspond to quantities directly measurable without any assumption on the behaviour of the angular distribution. This fact has two consequences: on the one hand, it prevents the nice test on the actual structure of the cross section which in the s-channel processes comes from the comparison of the directly measured parameters versus their fitted values. On the other hand, since their meaning for our process is connected to a Born-like description, there is no way of predicting them using full Standard Model calculations and therefore, their final interpretation requires the use of the same sort of approximations used for their extraction, which is something that does not occur in the s-channel processes.

- Furthermore, the disconnection between this parameters and the direct measurements is the origin of strong correlations (of the order of $80 \%$ ) among them when fitting the data. This causes an important technical problem in their interpretation and in their practical use: every single parameter measurement is useless if not considered in conjunction with the others and their complete correlation matrix. This means that, in fact, for every energy point, the set of number to be given to collapse the angular distribution information are: four measurements with their four errors plus six parameters giving their correlations. 
To overcome this problem, a reparameterization seems to be mandatory. The goal is finding a set of parameters which can be directly measured without any further assumption and computing their connection with the set of physical parameters that we have introduced when we assume the frame in which this parameter set is meaningful.

To simplify the problem at this stage, we will reduce the set of physical parameters to three by fixing the coefficient of the pure photon t-channel exchange term to its QED prediction. This is not really a lose of generality in the sense that, in our "model independent" approach, QED is taken as one of the building blocks (for instance to include photonic corrections) and, this is the only assumption we need to compute $A_{\gamma t}(s)$.

Since we have three physical quantities we need three measurements to obtain them univocally. Two of them which do not need further justification are the total integrated cross section within the kinematical cuts (in $c \equiv \cos \theta$ and $Y$ )

$$
\sigma=\int_{c_{-}}^{c_{+}} d \sigma
$$

and the total forward-backward charge asymmetry within the same cuts

$$
A_{F B}=\frac{\sigma_{F}-\sigma_{B}}{\sigma}=\frac{1}{\sigma}\left(\int_{0}^{c_{+}}-\int_{c_{-}}^{0}\right) d \sigma=1-2 \frac{\int_{c_{-}}^{0} d \sigma}{\sigma}
$$

For the third one we have chosen a center-edge charge asymmetry defined in the following way:

$$
A_{C E}=\frac{1}{\sigma}\left(\int_{0.5}^{c_{+}}+\int_{c_{-}}^{-0.5}-\rho \int_{-0.5}^{0.5}\right) d \sigma=1-(1+\rho) \frac{\int_{-0.5}^{0.5} d \sigma}{\sigma}
$$

where $\rho$ is a numerical factor defined as

$$
\rho \equiv \frac{\left(\int_{0.5}^{c_{+}}+\int_{c_{-}}^{-0.5}\right)\left(1+c^{2}\right) d c}{\int_{-0.5}^{0.5}\left(1+c^{2}\right) d c}
$$

so that this observable vanishes exactly for angular distributions of the type of $1+c^{2}$ and $c$ and therefore its expectation is zero for a pure s-channel process. Therefore, this observable measures directly the non-s-channel component of the angular distribution.

In order to reparameterize the angular distribution built in terms of physical observables to one in terms of this measurable quantities, we just have to compute a conversion matrix relating both parameterizations. This can be done just by recalling that what we have built is

$$
\frac{d \sigma}{d c}=\sigma^{s}(s) f_{1}(c)+\sigma^{s}(s) A_{F B}^{s}(s) f_{2}(c)+A_{\text {int }}(s) f_{3}(c)+f_{4}(c)
$$

and then we have the simple relation

$$
\begin{aligned}
\sigma(s) & =\sigma^{s}(s) a_{11}+\sigma^{s}(s) A_{F B}^{s}(s) a_{12}+A_{\text {int }}(s) a_{13}+a_{14} \\
\sigma(s) A_{F B}(s) & =\sigma^{s}(s) a_{21}+\sigma^{s}(s) A_{F B}^{s}(s) a_{22}+A_{\text {int }}(s) a_{23}+a_{24} \\
\sigma(s) A_{C E}(s) & =\sigma^{s}(s) a_{31}+\sigma^{s}(s) A_{F B}^{s}(s) a_{32}+A_{\text {int }}(s) a_{33}+a_{34}
\end{aligned}
$$

being

$$
a_{1 i}=\int_{c_{-}}^{c_{+}} f_{i}(c) d c
$$




$$
\begin{aligned}
& a_{2 i}=\left(\int_{0}^{c_{+}}-\int_{c_{-}}^{0}\right) f_{i}(c) d c \\
& a_{3 i}=\left(\int_{0.5}^{c_{+}}+\int_{c_{-}}^{-0.5}-\rho \int_{-0.5}^{0.5}\right) f_{i}(c) d c
\end{aligned}
$$

coefficients which do not depend on the parameters. For $i=1,2,3$ they come from integrating $1+c^{2}, c$ and $\frac{\alpha\left(-\frac{1}{2} M_{Z}^{2}(1-c)\right)}{\alpha\left(M_{Z}^{2}\right)} \frac{\left(1+c^{2}\right)}{(c-1)}$ respectively, so that they are just constants. For $i=4$, they have a small dependence on $s$ coming from the $A_{\gamma t}$ and $\left(\frac{d \hat{\sigma}}{d \cos \theta}\right)_{i Z_{t}}$ terms. Table 1 shows the conversion matrix for $s=M_{Z}^{2}$. Inverting this matrix to substitute the physical parameters in

\begin{tabular}{|c||c|c|c|c|}
\hline$a_{1 i}$ & 0.8573 & 0 & -6.3078 & 0.5674 \\
\hline$a_{2 i}$ & 0 & 0.81 & -5.7645 & 0.5366 \\
\hline$a_{3 i}$ & 0 & 0 & -3.3816 & 0.4420 \\
\hline
\end{tabular}

Table 1: Conversion matrix for $c_{+}=-c_{-}=0.9$ and $|Y|<0.3$ at $\sqrt{s}=91.238 \mathrm{GeV}$.

equation (21) by the parameters directly measurable, it is straightforward to write a fitting formula for the angular distribution in terms of $\sigma, A_{F B}$ and $A_{C E}$. Using this reparameterized formula, this three parameters can be fit to the data and, as expected, their correlation turns out to be negligeable. Therefore, the relevant information for every energy point can be collapsed in just three parameters which are, in fact, independent measurements.

\subsection{Checks of the approach}

The accuracy of this formula has been checked by fitting a binned angular distribution produced with the ALIBABA program (see figure 8). Assuming errors in the ALIBABA prediction corresponding to half a million Bhabha events, the $\chi^{2}$ of the fit per degree of freedom, is of $4 / 15$ (basically coming from the statistical precision of the Monte Carlo integration used in ALIBABA, as can be seen in the figure). Besides, the actual quality of the angular distribution parameterization can be judged by comparing the fit parameters with the ones obtained directly from the data. As can be seen in figures 9, the fit parameters agree within better than 1 per mil with their direct theoretical predictions across the resonance for $|\cos \theta|<0.9$ and $|Y|<0.3$.

Since most of the available (and future) LEP luminosity is concentrated in one point, we show in table 2 the detailed comparison for a realistic "peak" energy. In this table, it can be seen that the gain in statistical error obtained by fitting the parameters instead of measuring them directly can represent as much as having $60 \%$ more statistics for $A_{C E}$ for instance.

\begin{tabular}{|c|c|c|c|}
\hline Parameter & Measured & Fitted & $\Delta$ \\
\hline \hline$\sigma$ & $1.8407 \pm 0.0026$ & $1.8406 \pm 0.0026$ & 0.03 \\
\hline$A_{F B}$ & $0.2953 \pm 0.0014$ & $0.2954 \pm 0.0013$ & -0.06 \\
\hline$A_{C E}$ & $0.2387 \pm 0.0014$ & $0.2388 \pm 0.0011$ & -0.04 \\
\hline
\end{tabular}

Table 2: Fit to the ALIBABA binned angular distribution for $M_{Z}=91.187 \mathrm{GeV}, m_{\text {top }}=140$ $\mathrm{GeV}, M_{\text {Higgs }}=300 \mathrm{GeV}$ and $\alpha_{s}=0.12$ for $|\cos \theta|<0.9$ and $|Y|<0.3$ at $\sqrt{s}=91.238 \mathrm{GeV}$. The errors correspond to half a million $e^{+} e^{-}$events. The $\Delta$ column is the difference in units of the measured error. 


\subsection{Extraction of electroweak parameters}

The last step in the use of the $e^{+} e^{-}$angular distribution is the interpretation of the measured parameters in terms of $Z$ parameters. LEP experiments do this at present by performing a combined fit of the cross section and forward-backward asymmetry for each separable final state $Z$ decay to a "model-independent" description in terms of the $Z$ effective parameters [13].

In order to enable this process for the new measurements, we need a calculation able to predict these measurements in terms of the $Z$ parameters and able to incorporate the cuts in the variables that we are using.

For the integrated cross section, the program MIBA exists [9] and fulfills completely our requirements. For the other two variables, $A_{F B}$ and $A_{C E}$, starting from the MIBA approach, we have applied minor refinements to the treatment of the effective couplings ${ }^{5}$.

The basic modifications that we have implemented consist just in applying a definition of the effective couplings following the substitutions of equation (8) which, for the case of the s-channel processes, produce a very accurate description of the couplings. The new MIBA, is able to produce very accurate predictions for these observables and, in fact, also for the binned angular distribution and its speed enables its use as a fitting formula. Figures 10 show the agreement between ALIBABA and MIBA for the three quantities introduced, as a function of the center-of-mass energy. The ALIBABA predictions have been computed for $M_{Z}=91.187$ $\mathrm{GeV}, m_{\text {top }}=140 \mathrm{GeV}, M_{\text {Higgs }}=300 \mathrm{GeV}$ and $\alpha_{s}=0.12$. The MIBA predictions are for $M_{Z}=91.187 \mathrm{GeV}, \Gamma_{Z}=2.4917 \mathrm{GeV}, \Gamma_{e}=83.69 \mathrm{MeV}$ and $\sin ^{2} \theta_{\text {eff }}^{\text {lept }}=0.2325$. These three last parameters are predicted by the electroweak ALIBABA library. Furthermore, as before, we give a more detailed comparison for a "peak" energy in table 3 .

\begin{tabular}{|c|c|c|c|}
\hline Parameter & ALIBABA & MIBA & \multicolumn{1}{|c}{} \\
\hline \hline$\sigma$ & 1.8399 & 1.8388 & $\delta=0.06$ \\
\hline$A_{F B}$ & 0.2984 & 0.2981 & $\Delta=0.03$ \\
\hline$A_{C E}$ & 0.2435 & 0.2429 & $\Delta=0.06$ \\
\hline
\end{tabular}

Table 3: Comparison between the leading log QED corrected ALIBABA and MIBA predictions for $\sigma, A_{F B}, A_{C E}$ at $\sqrt{s}=91.238 \mathrm{GeV}$ for $|\cos \theta|<0.9$ and $|Y|<0.3$. $\delta(\Delta)$ denotes the relative (absolute) difference in per cent.

\section{$6 \quad$ Missing photonic corrections}

In the whole previous study one of the basic assumptions implicit in our treatment is the feasibility of introducing photonic corrections in our calculations by just convoluting the s-dependent quantities with some kind of radiator functions without distorting sizably the $\cos \theta$ dependence of each contribution. This is based upon the image of the structure function approach, which we have implemented as in ALIBABA. Certainly this is an approximation whose validity has to be checked with a full calculation of the QED corrections.

So far, complete calculation of QED corrections for $e^{+} e^{-}$exist just up to $O(\alpha)$ and are incorporated in the ALIBABA program. In that program, QED corrections are splitted among

\footnotetext{
${ }^{5}$ In the original MIBA approach, an accurate treatment of the terms proportional to $v_{e}$ was not applied since they play an irrelevant role in the integrated total cross section.
} 
leading-log "LL" and non-leading-log "NLL". It is important to point out that, in spite that for each order in $\alpha$ the sum of both combinations is uniquely defined, their splitting is not unique since it depends on what is defined as "LL" contribution in a calculation. Since in MIBA we incorporate the "LL" corrections in the same way as in ALIBABA, we can evaluate the missing correction just by looking at the "NLL" corrections in ALIBABA. This corrections are typically much smaller than $1 \%$ in the binned angular distribution and they do not depend sizably on the actual value of the physical parameters that we want to measure. Therefore, we can compute them for each energy using ALIBABA and afterwards apply them as a small correction factor to the parameters:

$$
X(s)=X_{L L}(s)(1+\Delta X(s)) \quad X \equiv \sigma, A_{F B} \text { and } A_{C E}
$$

Table 4 shows the size of this corrections for $\sigma, A_{F B}$ and $A_{C E}$ for different center-of-mass energies.

\begin{tabular}{|c|ccccccc|}
\hline$\sqrt{s}(\mathrm{GeV})$ & 88.238 & 89.238 & 90.238 & 91.238 & 92.238 & 93.238 & 94.238 \\
\hline$\Delta \sigma$ & -0.32 & -0.35 & -0.20 & -0.06 & -0.14 & -0.24 & -0.35 \\
\hline$\Delta A_{F B}$ & -0.33 & -0.27 & -0.49 & -1.00 & -1.26 & -0.95 & -0.47 \\
\hline$\Delta A_{C E}$ & -0.24 & -0.49 & -0.77 & -1.45 & -1.55 & -1.08 & -0.77 \\
\hline
\end{tabular}

Table 4: The non-leading-log contribution (in per cent) at LEP energies for $|\cos \theta|<0.9$ and $|Y|<0.3$.

\section{Conclusions}

We have shown that the analysis of the complete angular distribution of the process $e^{+} e^{-} \rightarrow e^{+} e^{-}$ is needed to fully exploit the physical information that it contains. Motivated by this fact, we have presented two different approaches enabling this possibility. One of them is inspired in the procedure presently applied in the treatment of the s-channel processes and hence can be easily integrated in the present analysis scheme.

We have compared our approaches with the most accurate existing predictions and the conclusion is that their accuracy is more than enough for statistics far beyond the ones LEP is expected to produce in the future.

The FORTRAN code implementing the approaches described in this paper can be be directly obtained from COMAS@CERNVM or MARTINEZ@CERNVM. 


\section{Appendix: Calculation of sensitivity functions}

Three different sensitivity functions have been built to handle the three different approaches discussed in the text.

(1) In the first case $A_{F B}$ is thought to be a function of only $A_{F B}^{0}$, that is,

$$
A_{F B}=\frac{\sigma_{F}-\sigma_{B}}{\sigma_{F}+\sigma_{B}}=A_{F B}\left(A_{F B}^{0}\right)
$$

where $\sigma_{F}$ and $\sigma_{B}$ are the forward and backward cross sections. Therefore, we have

$$
\Delta A_{F B}^{0}=\left(\frac{d A_{F B}}{d A_{F B}^{0}}\right)^{-1} \Delta A_{F B}
$$

The error in the forward-backward asymmetry can be written as

$$
\Delta A_{F B}=\frac{2}{\sqrt{L}} \sqrt{\frac{\sigma_{F} \sigma_{B}}{\sigma^{3}}}
$$

where $L$ denotes the luminosity accumulated during the study of the process. From these two equations we can write

$$
\Delta A_{F B}^{0}=\frac{1}{\sqrt{L}}\left(\frac{d A_{F B}}{d A_{F B}^{0}}\right)^{-1} 2 \sqrt{\frac{\sigma_{F} \sigma_{B}}{\sigma^{3}}}=\frac{1}{\sqrt{L}} \frac{1}{S_{A_{F B}}\left(A_{F B}^{0}\right)}
$$

Thus, we have disentangled the statistical sensitivity of $\Delta A_{F B}^{0}(1 / \sqrt{L})$ from its dependence on the functionality of the forward-backward asymmetry as a function of $A_{F B}^{0}$. This functionality is given by the inverse of the sensitivity function $S_{A_{F B}}\left(A_{F B}^{0}\right)$, defined as:

$$
S_{A_{F B}}\left(A_{F B}^{0}\right) \equiv \frac{1}{2} \sqrt{\frac{\sigma^{3}}{\sigma_{F} \sigma_{B}}} \frac{d A_{F B}}{d A_{F B}^{0}}
$$

(2) In the second approach $A_{F B}$ depends also on $\Gamma_{e}$, that is,

$$
A_{F B}=A_{F B}\left(A_{F B}^{0}, \Gamma_{e}\right)
$$

and, of course,

$$
\sigma=\sigma\left(A_{F B}^{0}, \Gamma_{e}\right)
$$

Assuming a non-singular Jacobian, we can invert this relations to obtain

$$
\begin{aligned}
A_{F B}^{0} & =A_{F B}^{0}\left(A_{F B}, \sigma\right) \\
\Gamma_{e} & =\Gamma_{e}\left(A_{F B}, \sigma\right)
\end{aligned}
$$

Then, applying proper error propagation,

$$
\begin{aligned}
\Delta^{2} A_{F B}^{0} & =\left(\frac{\partial A_{F B}^{0}}{\partial A_{F B}}\right)^{2} \Delta^{2} A_{F B}+\left(\frac{\partial A_{F B}^{0}}{\partial \sigma}\right)^{2} \Delta^{2} \sigma \\
& +\frac{1}{2} \rho \frac{\partial A_{F B}^{0}}{\partial A_{F B}} \frac{\partial A_{F B}^{0}}{\partial \sigma} \Delta A_{F B} \Delta \sigma \\
\Delta^{2} \Gamma_{e} & =\left(\frac{\partial \Gamma_{e}}{\partial A_{F B}}\right)^{2} \Delta^{2} A_{F B}+\left(\frac{\partial \Gamma_{e}}{\partial \sigma}\right)^{2} \Delta^{2} \sigma \\
& +\frac{1}{2} \rho \frac{\partial \Gamma_{e}}{\partial A_{F B}} \frac{\partial \Gamma_{e}}{\partial \sigma} \Delta A_{F B} \Delta \sigma
\end{aligned}
$$


where $\rho \equiv \rho\left(A_{F B}, \sigma\right)$ is the experimental correlation between the measurement of $A_{F B}$ and $\sigma$. Since these measurements are uncorrelated, $\rho=0$, and, therefore, we can write,

$$
\left(\begin{array}{c}
\Delta^{2} A_{F B}^{0} \\
\Delta^{2} \Gamma_{e}
\end{array}\right)=\left(\begin{array}{c}
\left(\frac{\partial A_{F B}^{0}}{\partial A_{F B}}\right)^{2}\left(\frac{\partial A_{F B}^{0}}{\partial \sigma}\right)^{2} \\
\left(\frac{\partial \Gamma_{e}}{\partial A_{F B}}\right)^{2}\left(\frac{\partial \Gamma_{e}}{\partial \sigma}\right)^{2}
\end{array}\right)\left(\begin{array}{c}
\Delta^{2} A_{F B} \\
\Delta^{2} \sigma
\end{array}\right)
$$

If we recall,

$$
\begin{aligned}
\Delta A_{F B} & =\frac{2}{\sqrt{L}} \sqrt{\frac{\sigma_{F} \sigma_{B}}{\sigma^{3}}} \\
\Delta \sigma & =\sqrt{\frac{\sigma}{L}}
\end{aligned}
$$

we obtain the combined sensitivity functions,

$$
\begin{aligned}
S_{A_{F B} \sigma}\left(A_{F B}^{0}\right) & \equiv\left(4 \frac{\sigma_{F} \sigma_{B}}{\sigma^{3}}\left(\frac{\partial A_{F B}^{0}}{\partial A_{F B}}\right)^{2}+\sigma\left(\frac{\partial A_{F B}^{0}}{\partial \sigma}\right)^{2}\right)^{-\frac{1}{2}} \\
S_{A_{F B} \sigma}\left(\Gamma_{e}\right) & \equiv\left(4 \frac{\sigma_{F} \sigma_{B}}{\sigma^{3}}\left(\frac{\partial \Gamma_{e}}{\partial A_{F B}}\right)^{2}+\sigma\left(\frac{\partial \Gamma_{e}}{\partial \sigma}\right)^{2}\right)^{-\frac{1}{2}}
\end{aligned}
$$

Finally, to obtain the derivatives with respect to $A_{F B}$ and $\sigma$, we make use of equations $(35)$ and $(36)$

$$
\left.\left(\begin{array}{c}
\left(\frac{\partial A_{F B}^{0}}{\partial A_{F B}}\right)\left(\frac{\partial A_{F B}^{0}}{\partial \sigma}\right) \\
\left(\frac{\partial \Gamma_{e}}{\partial A_{F B}}\right)\left(\frac{\partial \Gamma_{e}}{\partial \sigma}\right)
\end{array}\right)=\left(\begin{array}{c}
\left(\frac{\partial A_{F B}}{\partial A_{F B}^{\circ}}\right)\left(\frac{\partial A_{F B}}{\partial \Gamma_{e}}\right) \\
\left(\frac{\partial \sigma}{\partial A_{F B}^{0}}\right)\left(\frac{\partial \sigma}{\partial \Gamma_{e}}\right)
\end{array}\right)\right)^{-1}
$$

as equations (37) are just the invers.

(3) Finally, a more general way of obtaining the sensitivity function of a parameter from any function of it, is the use of a $\chi^{2}$ minimization to get the error on the determination of that parameter. In our case, as

$$
\sigma=\sigma\left(A_{F B}^{0}, \Gamma_{e}\right)
$$

then

$$
S_{\sigma}\left(A_{F B}^{0}\right)=\frac{1}{\sqrt{L}} \frac{1}{\Delta A_{F B}^{0}}
$$

So if $L$ which accounts for the statistical sensitivity is taken, without loss of generality, as unity, the sensitivity of $A_{F B}^{0}$ is only related to its error.

The $\chi^{2}$ will be evaluated as,

$$
\chi^{2}\left(A_{F B}^{0}, \Gamma_{e}\right)=\left(\frac{\sigma\left(\left.A_{F B}^{0}\right|_{f i x e d},\left.\Gamma_{e}\right|_{f i x e d}\right)-\sigma\left(A_{F B}^{0}, \Gamma_{e}\right)}{\delta \sigma\left(A_{F B}^{0}, \Gamma_{e}\right)}\right)^{2}
$$

and

$$
\delta \sigma=\frac{\sqrt{N}}{L}=\sqrt{\sigma}
$$

as $L$ is taken to be the unity. Therefore, the minimization function is,

$$
\chi^{2}\left(A_{F B}^{0}, \Gamma_{e}\right)=\frac{\left(\sigma\left(\left.A_{F B}^{0}\right|_{f i x e d},\left.\Gamma_{e}\right|_{f i x e d}\right)-\sigma\left(A_{F B}^{0}, \Gamma_{e}\right)\right)^{2}}{\sigma\left(A_{F B}^{0}, \Gamma_{e}\right)}
$$


Of course, as we are interested in the most general analysis of the differential cross section, we'll make use of the whole angular distribution to get the integrated sensitivity function. So for an n-binned angular distribution,

$$
\chi^{2}\left(A_{F B}^{0}, \Gamma_{e}\right)=\sum_{i=1}^{n} \frac{\left(\sigma_{i}\left(\left.A_{F B}^{0}\right|_{f i x e d},\left.\Gamma_{e}\right|_{f i x e d}\right)-\sigma_{i}\left(A_{F B}^{0}, \Gamma_{e}\right)\right)^{2}}{\sigma_{i}\left(A_{F B}^{0}, \Gamma_{e}\right)}
$$

In the case of two bins $(n=2)$, we reproduce exactly the results obtained in the combined sensitivity approach.

\section{Acknowledgements}

We have benefit from many useful discussions with J. Lefrançois and R. Miquel. We want to thank them and also M. Delfino, H. Meinhard, F. Rivera and D. Schlatter for their interest and their comments on this manuscript. 


\section{References}

[1] D.Decamp et al. (ALEPH Coll.), Z. Phys. C-Particles and Fields 52, 1 (1992).

[2] L.Rolandi and R.Tanaka at the International Conference on High Energy Physics ICHEP 92, Dallas, U.S.A., August 1992.

[3] M. Böhm, A. Denner, W. Hollik, Nucl. Phys. B304 687 (1988).

[4] D. Bardin, W. Hollik, T. Riemann, Z. Phys. C-Particles and Fields 49, 485 (1991).

[5] W. Beenakker, F.A. Berends, S.C. van der Marck, Nucl. Phys. B349, 323 (1991). The version we have actually used allows cuts in $\cos \theta$ in the center-of-mass frame and rapidity and is called ALISTAR (W. Beenakker, M. Martinez and R. Miquel).

[6] M. Cacciari, G. Montagna, O. Nicrosini, G. Passarino, Phys. Lett.B279, 384 (1992) and "40THIEVES" package.

[7] M. Bilenky, "BHANG" package inside the "ZFITTER" library, D. Bardin et al. CERNTH.6443/92.

[8] F.A. Berends et al. in Z Physics at LEP I, G. Altarelli at al. (eds), CERN 89-08, 1989.

A. Borelli, M. Consoli, L. Maiani, R. Sisto, Nucl. Phys. B333, 357 (1990).

R.N. Cahn, Phys. Rev. D36 2666 (1987).

M. Martinez et al. Z. Phys. C-Particles and Fields 49645 (1991).

[9] M. Martinez, R. Miquel, Z. Phys. C-Particles and Fields 53, 115 (1992).

[10] Z. Wąs, S. Jadach, Phys. Rev. D41, 1425 (1990).

[11] P. Comas, Ph.D. thesis, in preparation.

[12] D. Levinthal, F. Bird, R.G. Stuart, B.W. Lynn, Z. Phys. C-Particles and Fields 53, 617 (1992).

[13] The LEP Collaborations: ALEPH, DELPHI, L3 and OPAL, Phys. Lett. B276, 247 (1992). 


\section{List of Figures}

1 Contribution of the different amplitudes squared and interferences to the Bhabha angular distribution at three different center-of-mass energies. a), c), e) $\mathrm{Ab}$ solute differential cross section. Upper full line: total differential cross section; dashed line: pure s-channel contribution; dotted line: $t$-channel photon amplitude squared; dash-dotted line: interference between s-channel $\mathrm{Z}$ and t-channel photon amplitudes; long dashed line: interference between s-channel and t-channel photon amplitudes; lower full line: t-channel $\mathrm{Z}$ contributions. b), d), f) Relative contribution in per cent. . . . . . . . . . . . . .

2 Sensitivity with respect to $A_{F B}^{0}$ as a function of $\cos \theta$ for the first approach followed in the text. a) At $3 \mathrm{GeV}$ below the $\mathrm{Z}$ peak. b) At the $\mathrm{Z}$ peak. c) At $3 \mathrm{GeV}$ above the $\mathrm{Z}$ peak. d) As a function of the center-of-mass energy for $|\cos \theta|<0.9$. The dashed line is completely superimposed to the full one because in this approach we are freezing completely the non-s-channel contribution. . . . . . . . . . .

3 Sensitivity with respect to $\Gamma_{e}$ as a function of the center-of-mass energy for $|\cos \theta|<0.9$ for the second approach followed in the text. . . . . . . .

4 Sensitivity with respect to $A_{F B}^{0}$ as a function of $\cos \theta$ for the second approach followed in the text. a) At $3 \mathrm{GeV}$ below the $\mathrm{Z}$ peak. b) At the $\mathrm{Z}$ peak. Since in a tree level calculation, the interference is 0 , the dashed line is completely superimposed to the full one. c) At $3 \mathrm{GeV}$ above the $\mathrm{Z}$ peak. d) As a function of the center-of-mass energy for $|\cos \theta|<0.9 \ldots \ldots \ldots \ldots$

5 Sensitivity with respect to $A_{F B}^{0}$ as a function of $\cos \theta$ for the third approach followed in the text. a) At $3 \mathrm{GeV}$ below the $\mathrm{Z}$ peak. b) At the $\mathrm{Z}$ peak. Since in a tree level calculation, the interference is 0 , the dashed line is completely superimposed to the full one. c) At $3 \mathrm{GeV}$ above the $\mathrm{Z}$ peak. d) As a function of the center-of-mass energy for $|\cos \theta|<0.9 \ldots \ldots \ldots \ldots \ldots$

6 Comparison between the leading $\log$ QED corrected angular distribution predicted by [5] and DMIBA at $\sqrt{s}=M_{Z}$. a) Angular distribution. The agreement is such that both lines appear almost superimposed. b) Relative difference in per cent. The dotted bars show the statistical uncertainty corresponding to half a million $e^{+} e^{-}$events. . . . . . . . . . . . . . . . .

7 Acollinearity versus $\cos \theta$ in the center-of-mass frame for different fractional energies left to the initial state electron after collinear radiation assuming that the positron did not radiate $\left(x_{+}=1\right)$. The correspoding rapidity values are also shown. 28

8 Comparison between the angular distribution predicted by [5] and our fitting formula at $\sqrt{s}=M_{Z}$. a) Angular distribution. The agreement is such that both lines appear almost superimposed. b) Relative difference in per cent. The dotted bars show the statistical uncertainty corresponding to half a million $e^{+} e^{-}$events.

9 Difference in per mil between the fit parameters predicted by [5] and our fitting formula in the $Z$ peak region for $|\cos \theta|<0.9$. The dotted bars show the statistical uncertainty corresponding to half a million $e^{+} e^{-}$events. a) Relative difference for $\sigma$. b) Absolute difference for $A_{F B}$. c) Absolute difference for $A_{C E} . \ldots$. . . 
10 Difference in per mil between the fit parameters predicted by [5] and MIBA with only leading $\log$ QED corrections in the $Z$ peak region for $|\cos \theta|<0.9$. The dotted bars show the statistical uncertainty corresponding to half a million $e^{+} e^{-}$ events. a) Relative difference for $\sigma$. b) Absolute difference for $A_{F B}$. c) Absolute

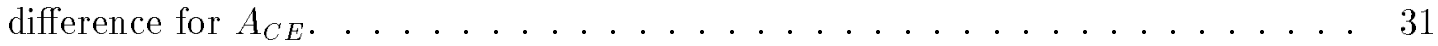




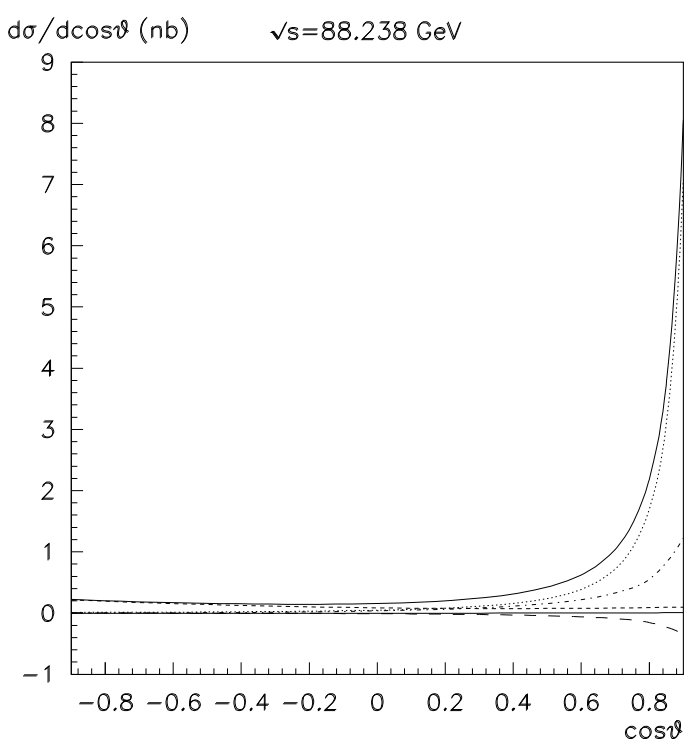

a)

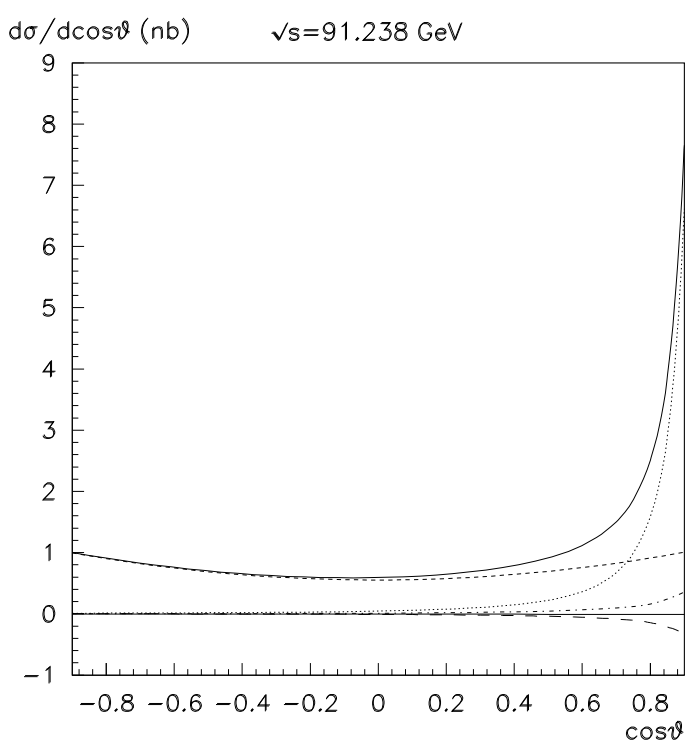

c)

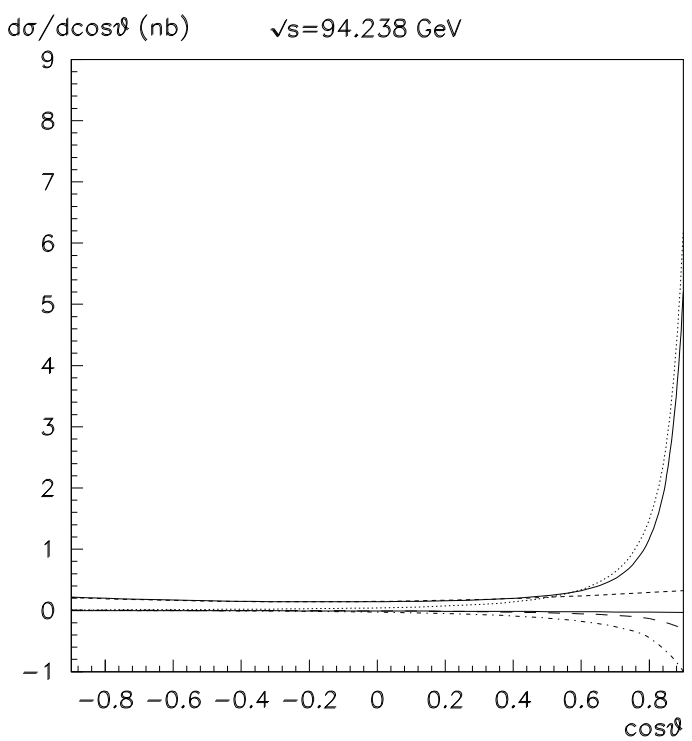

e)

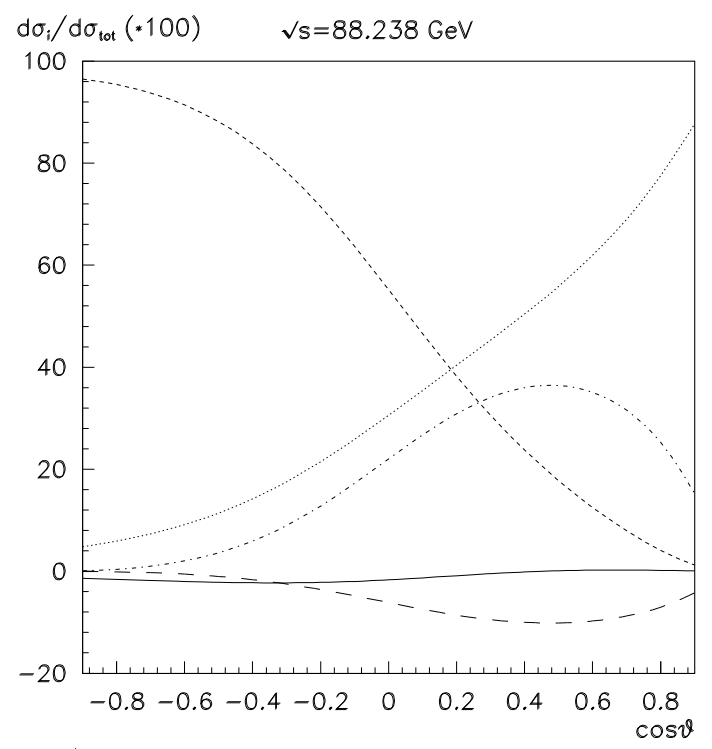

b)

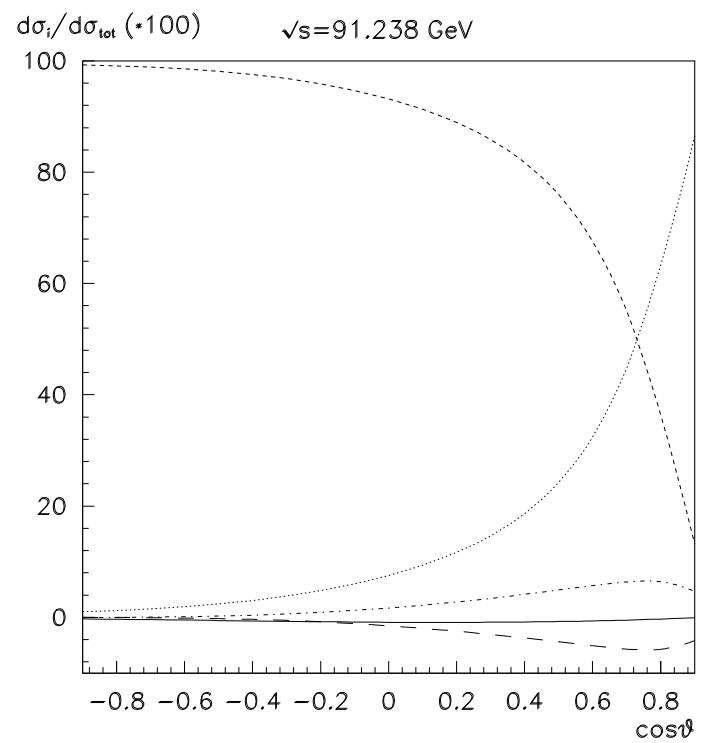

d)

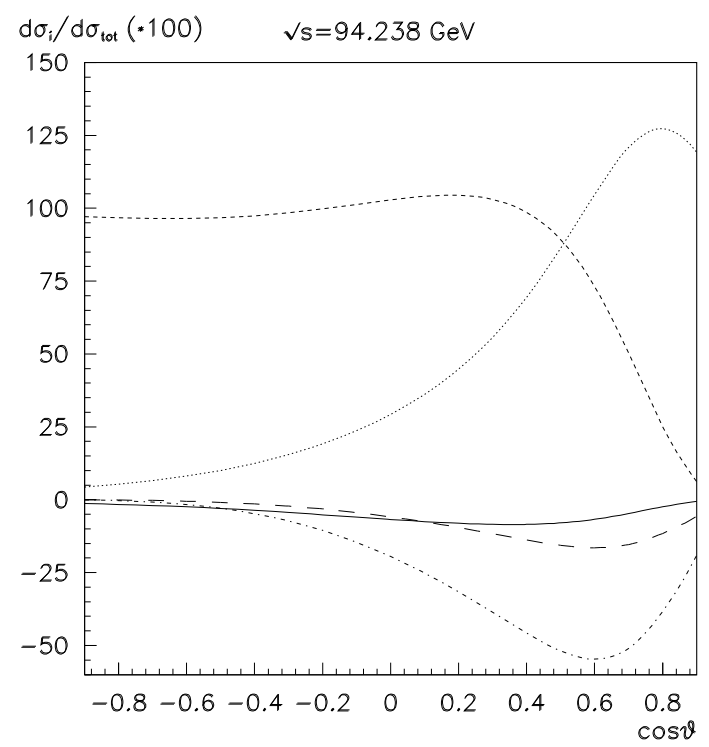

f)

Figure 1: 


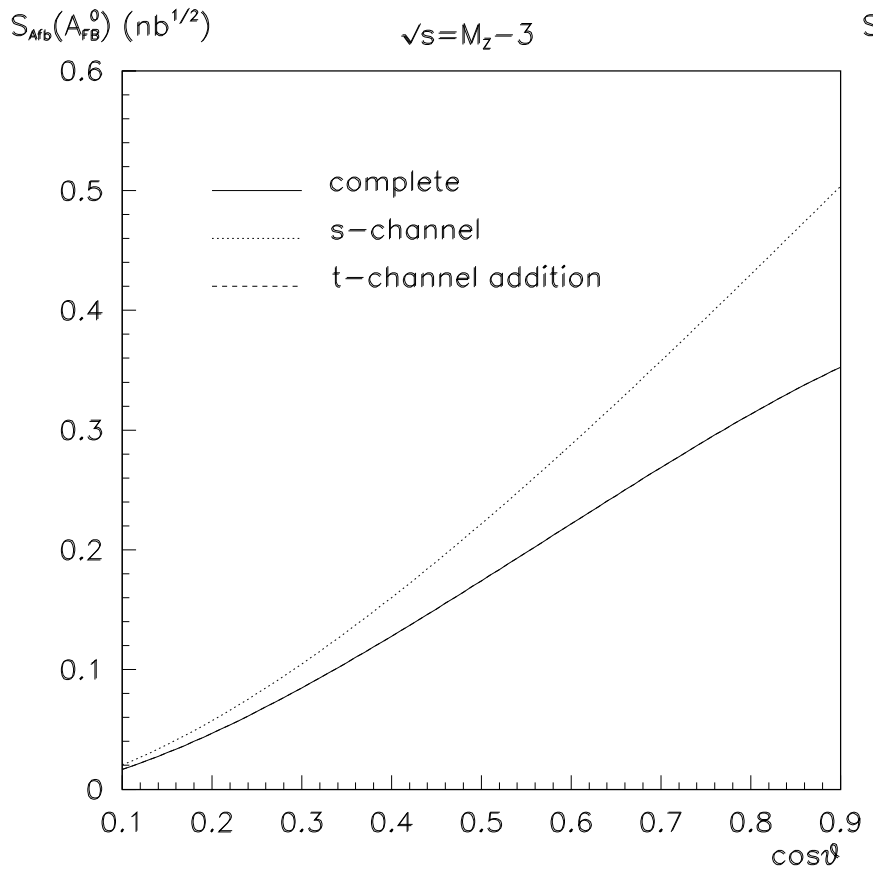

a)

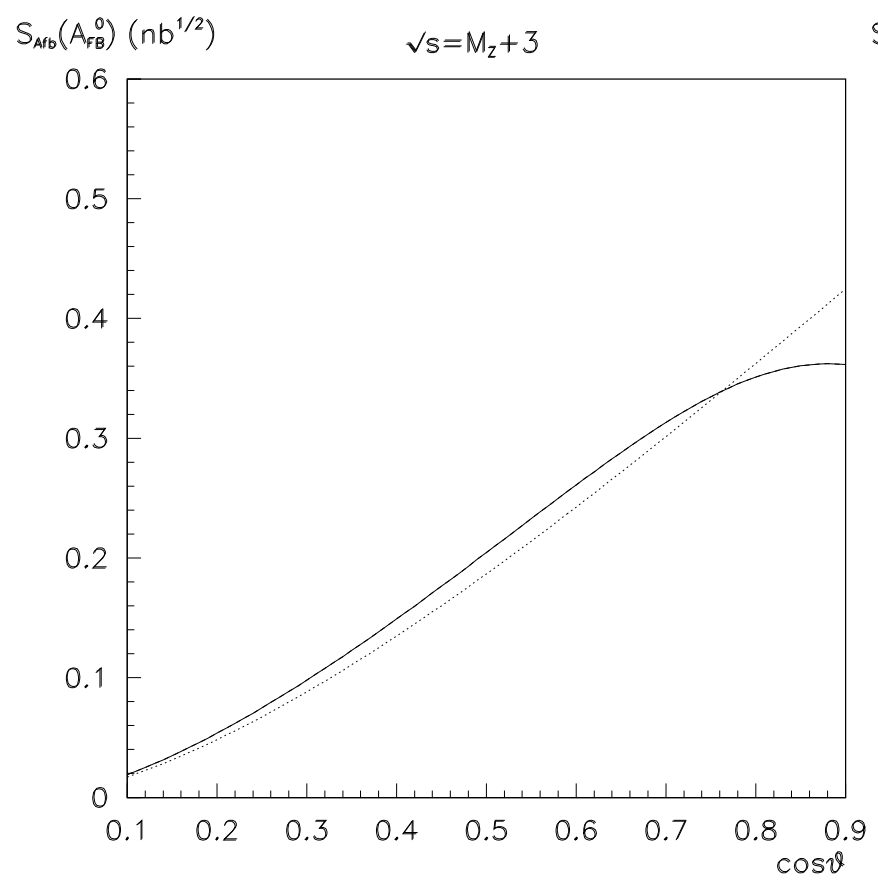

c)

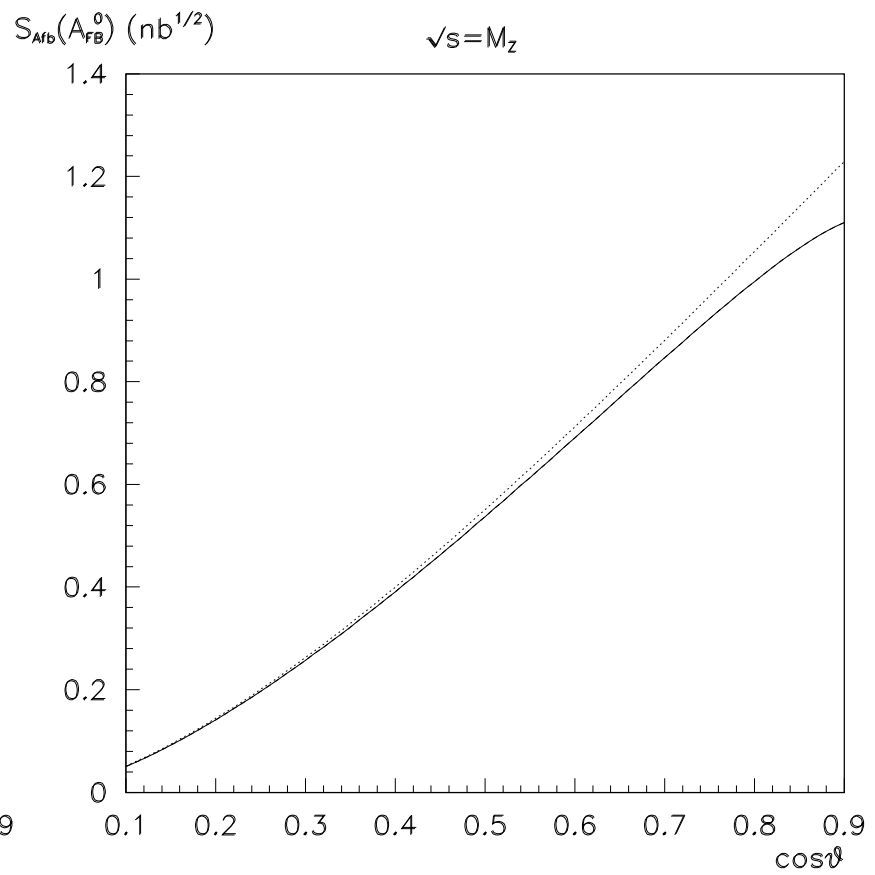

b)

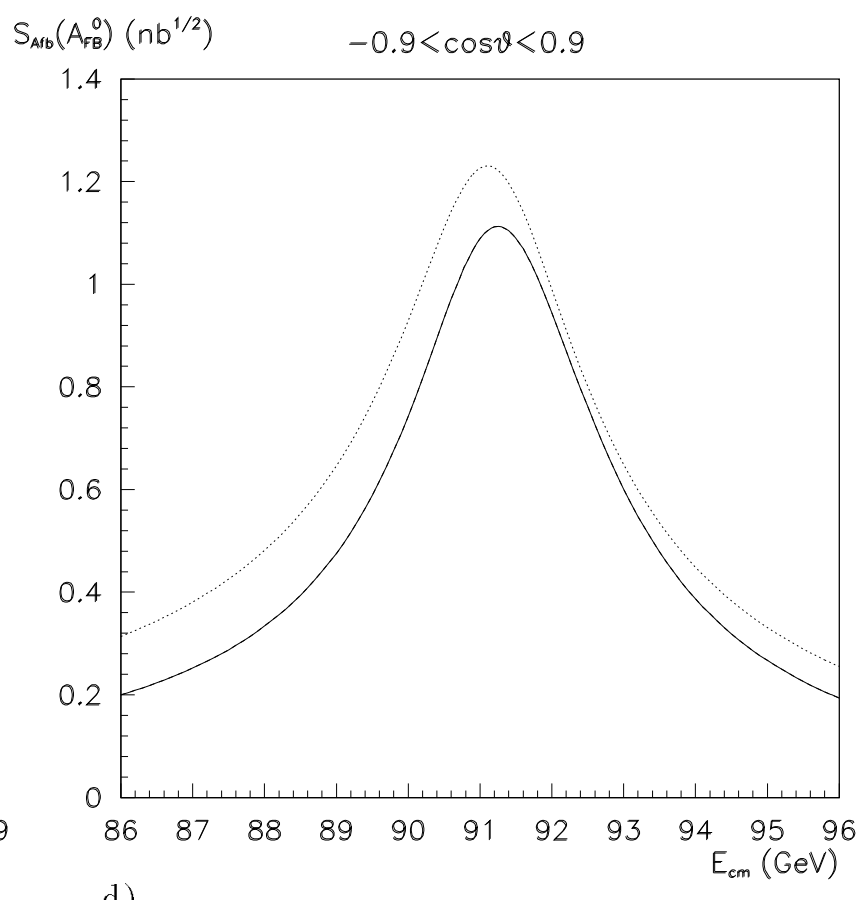

d)

Figure 2: 


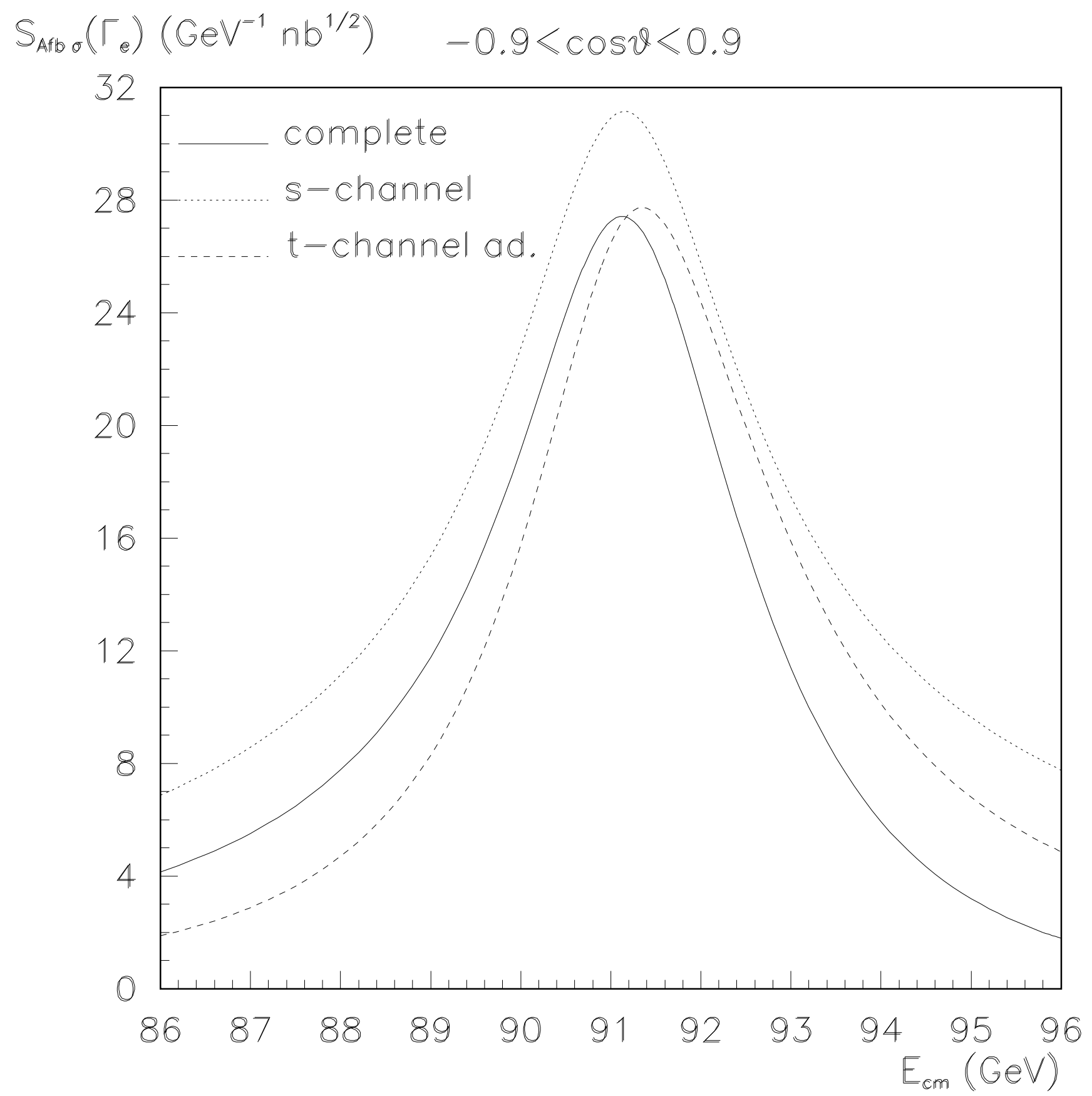

Figure 3: 


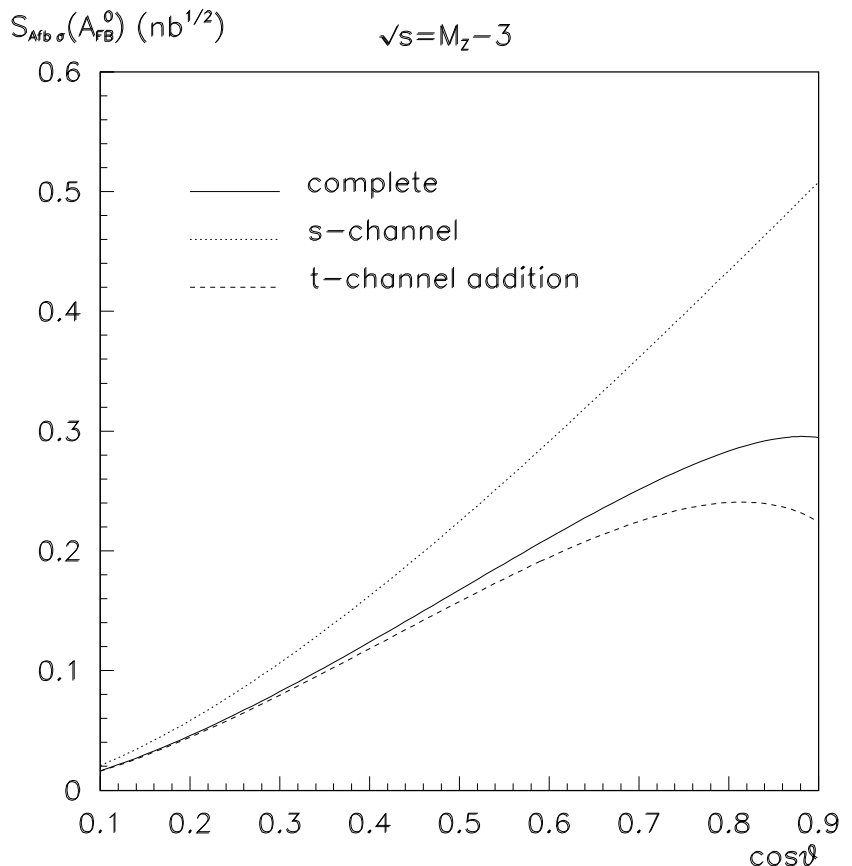

a)

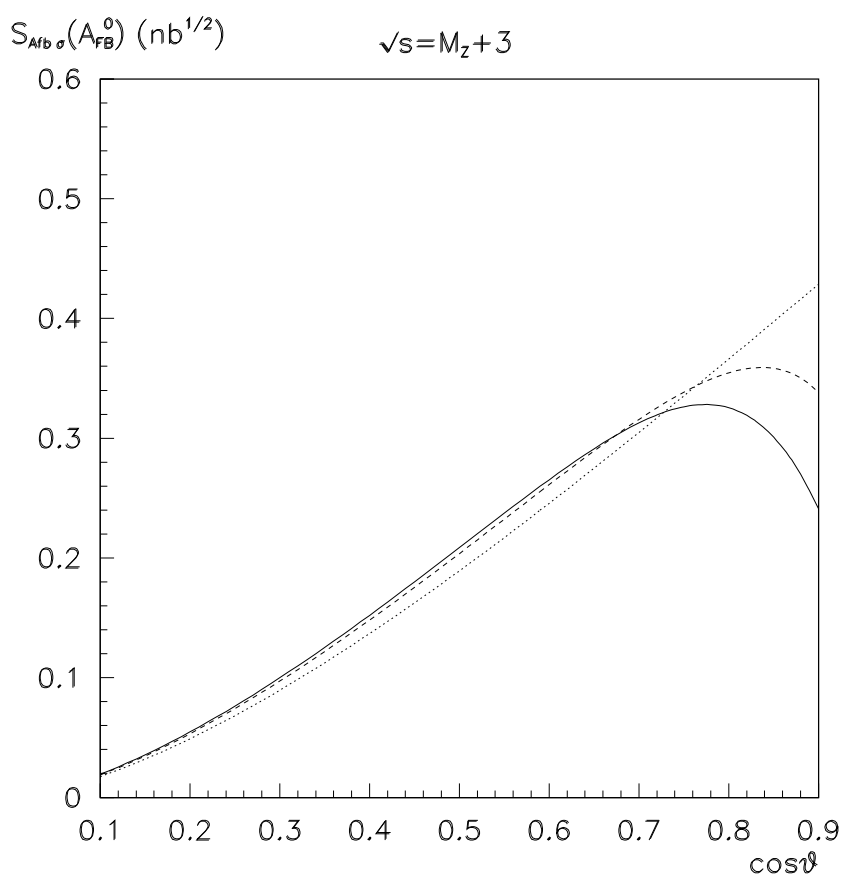

c)

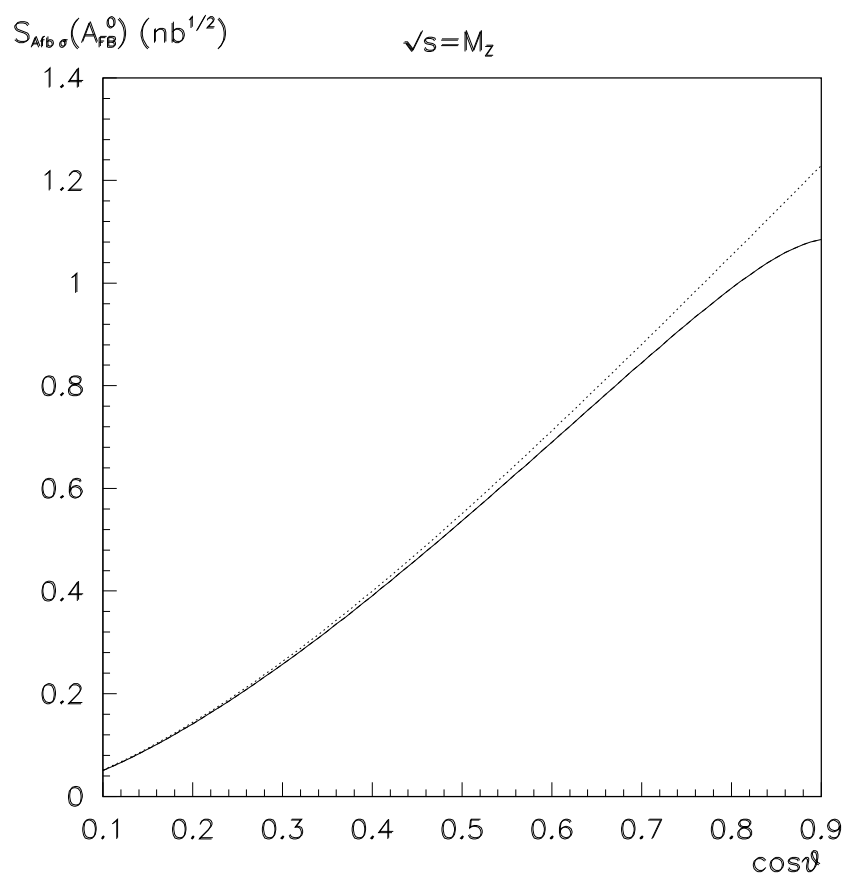

b)

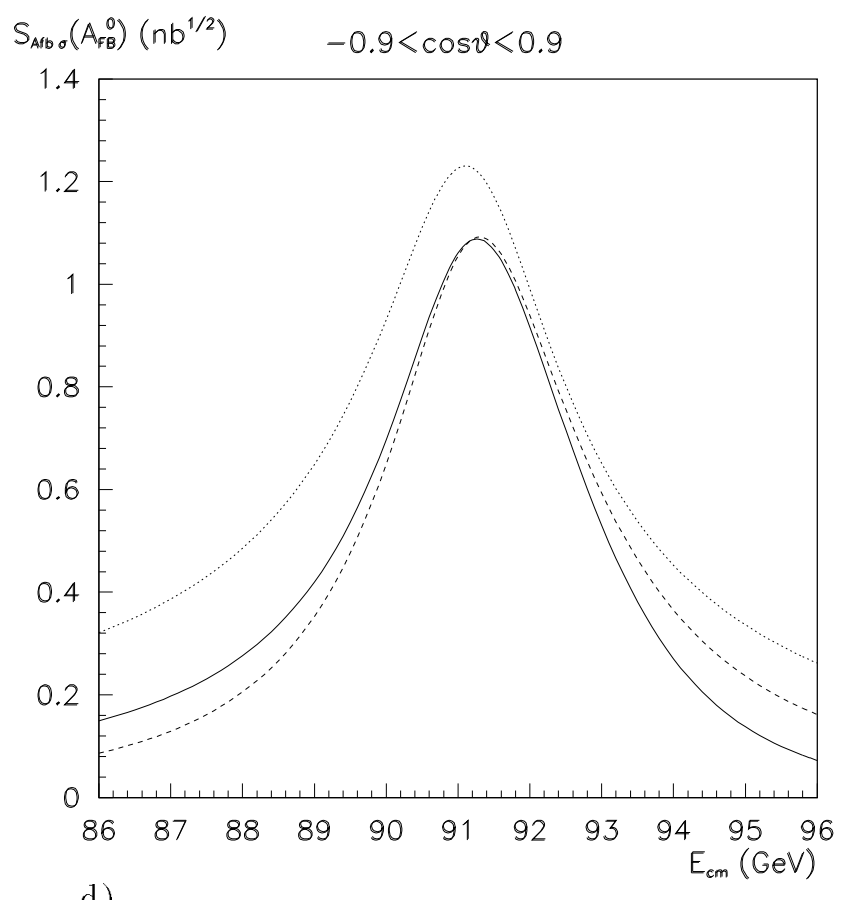

d)

Figure 4: 

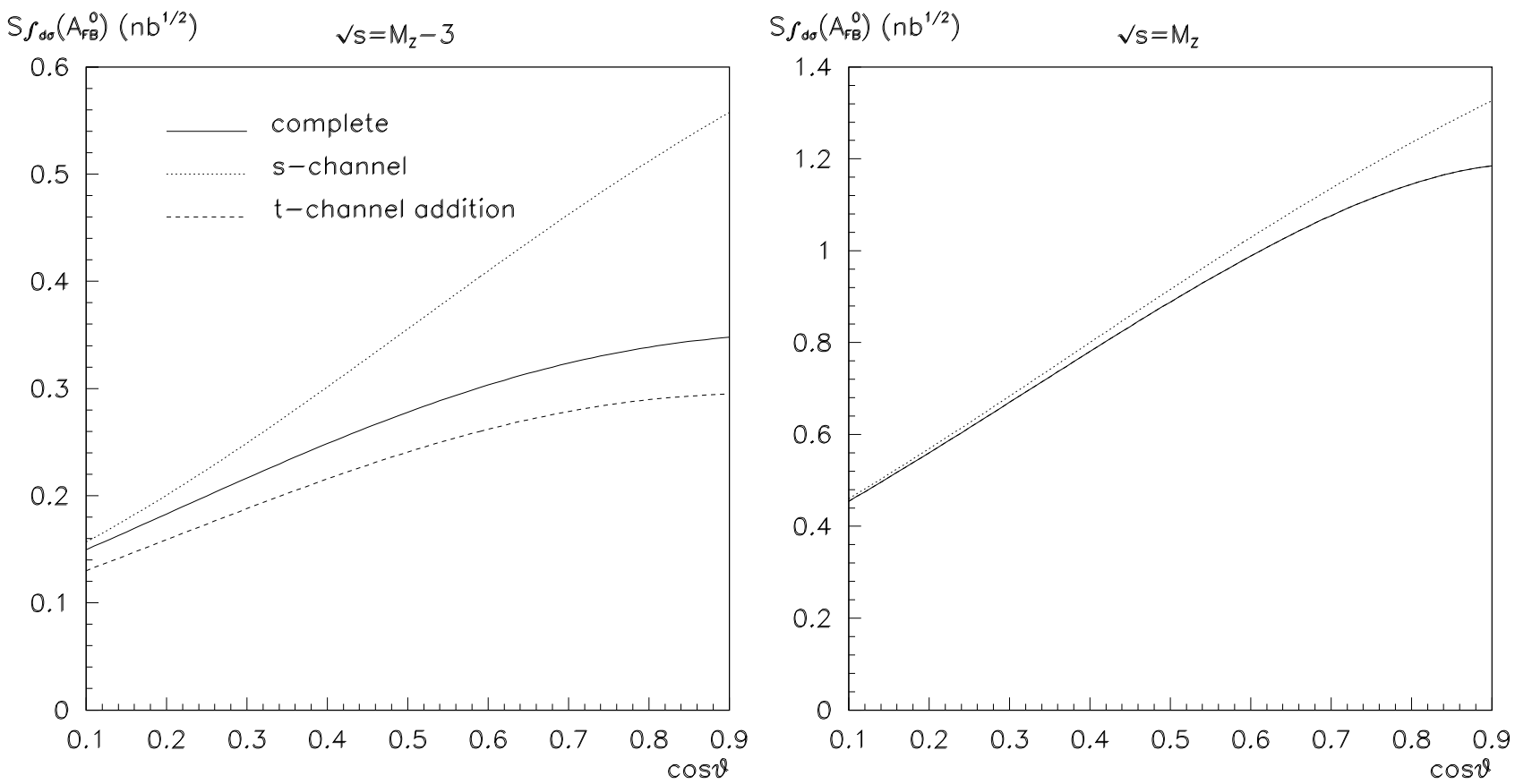

a)

b)

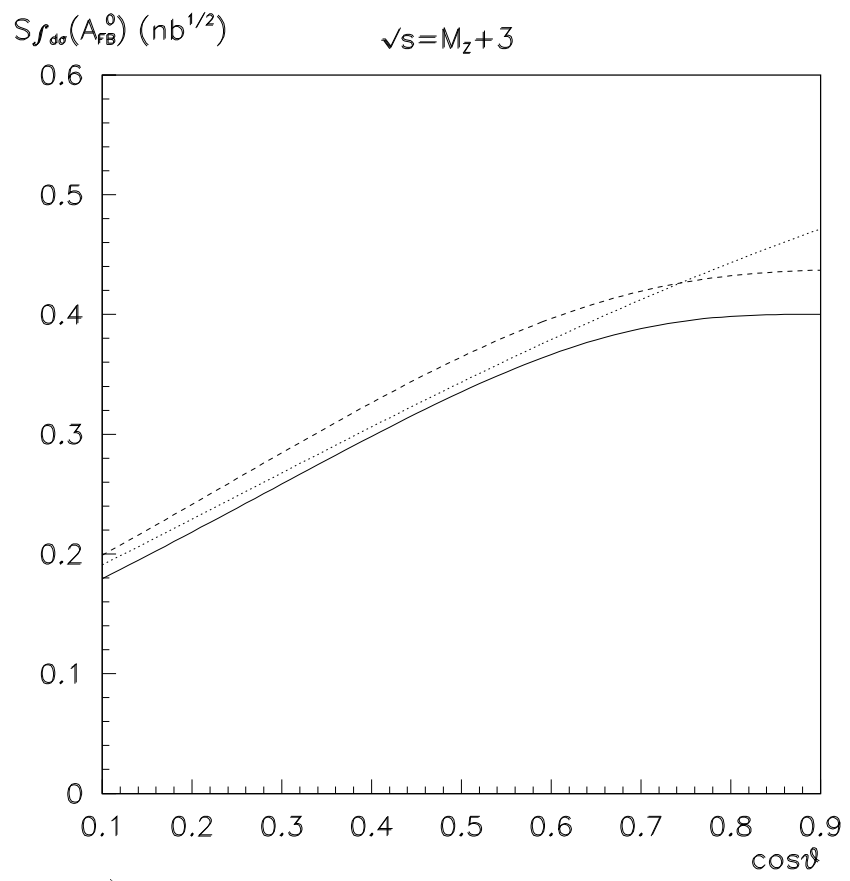

c)

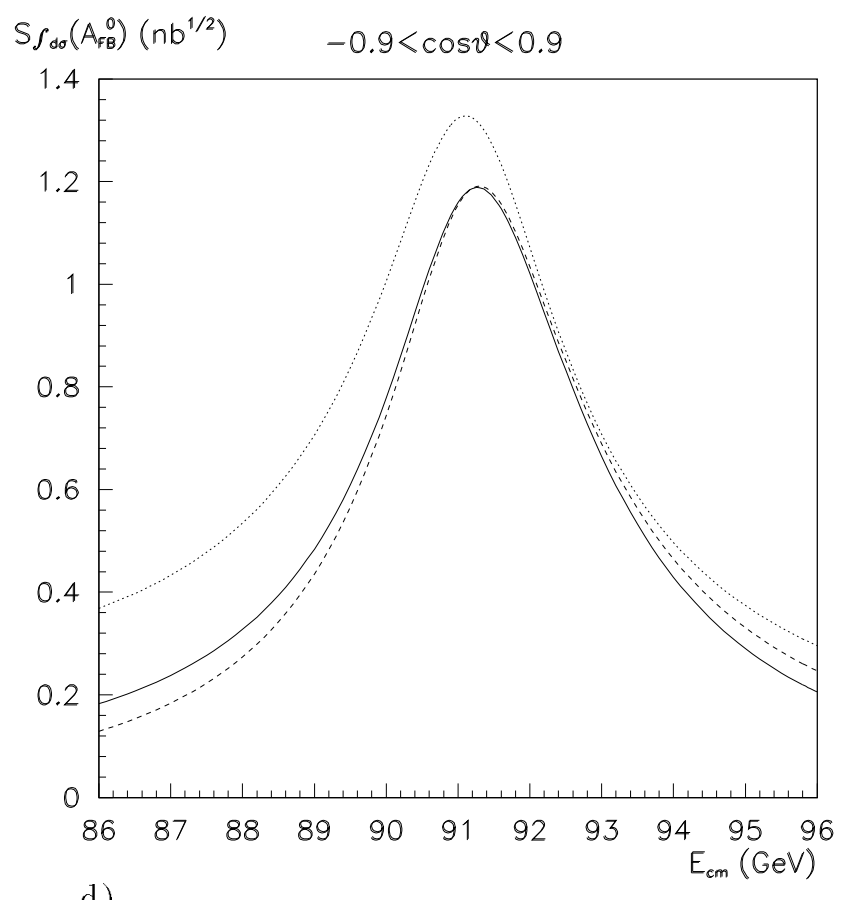

d)

Figure 5: 


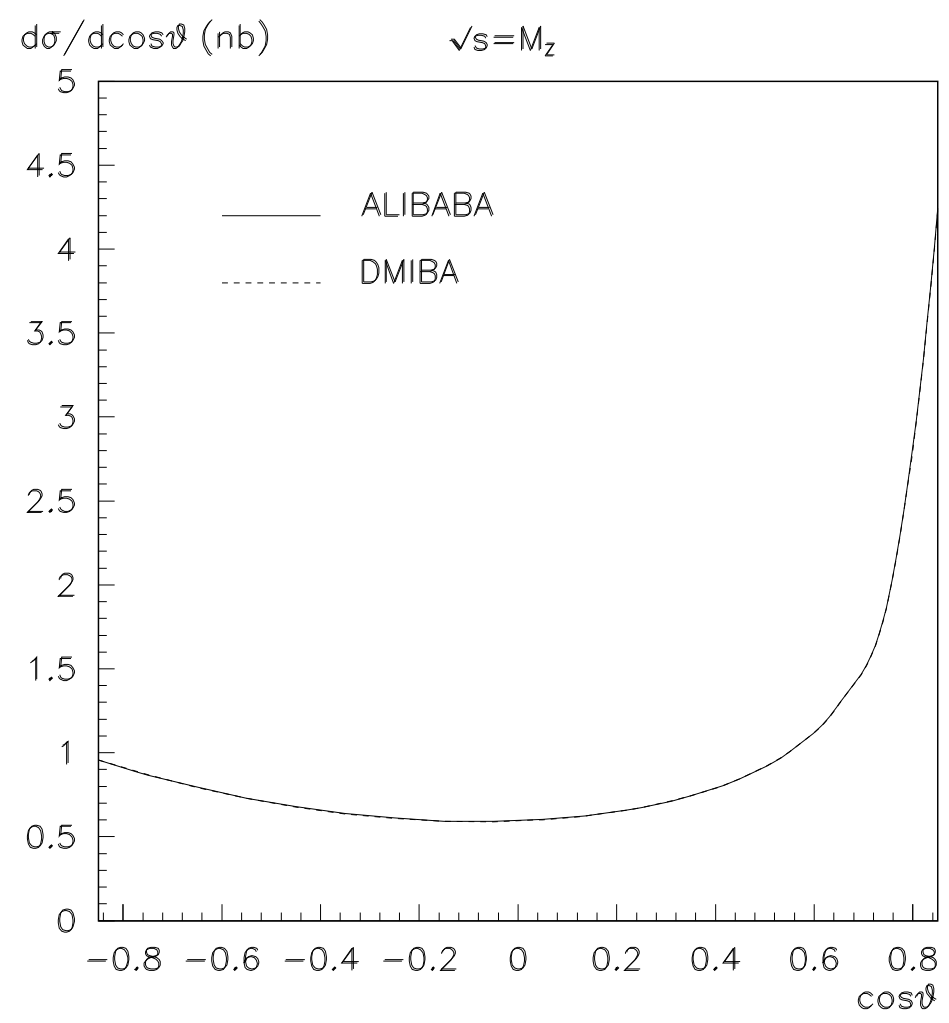

a)

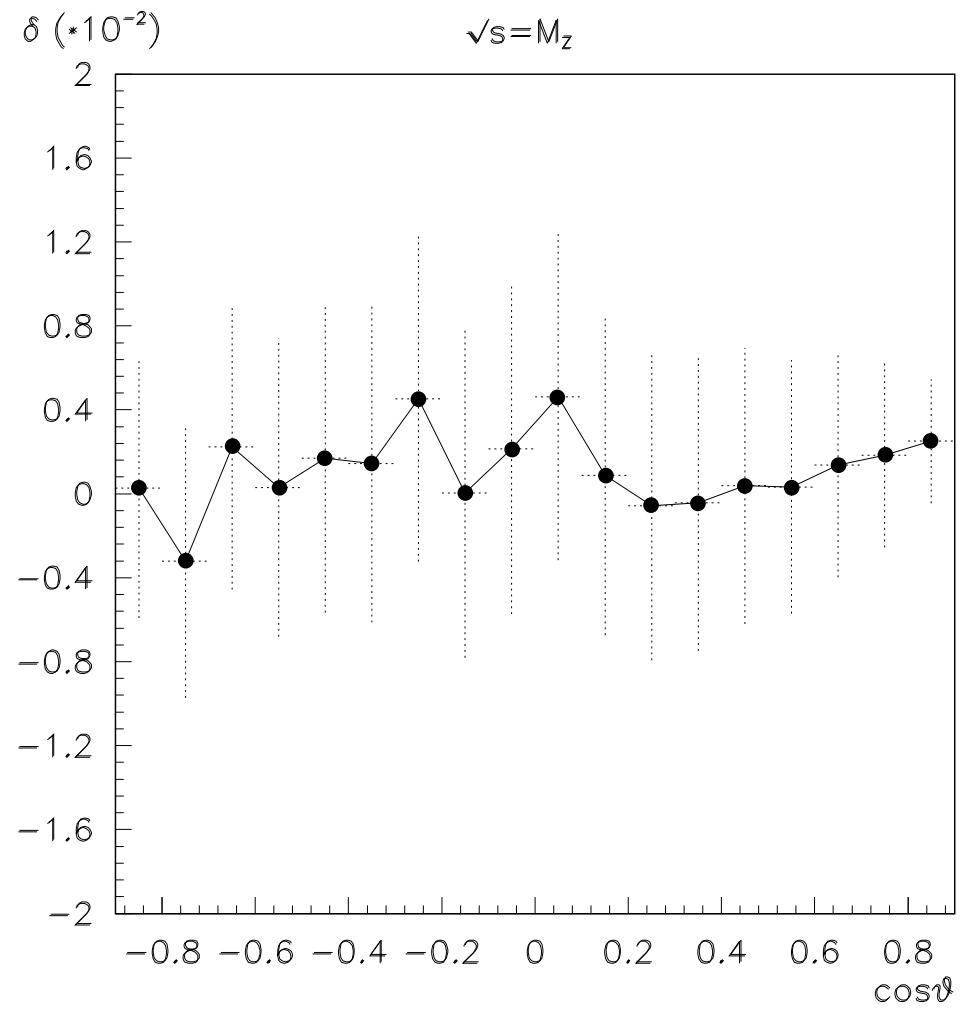

b)

Figure 6: 


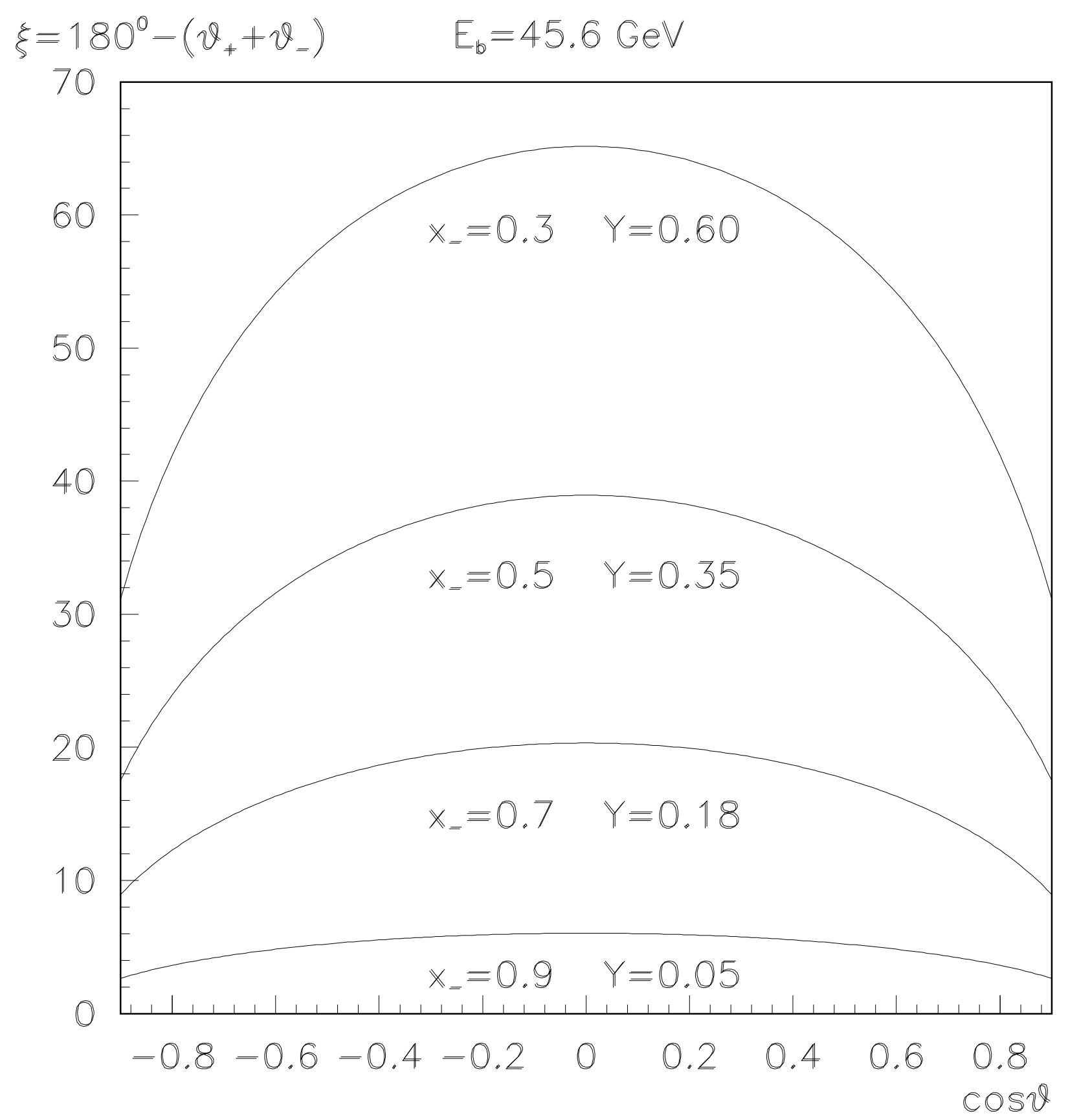

Figure 7: 


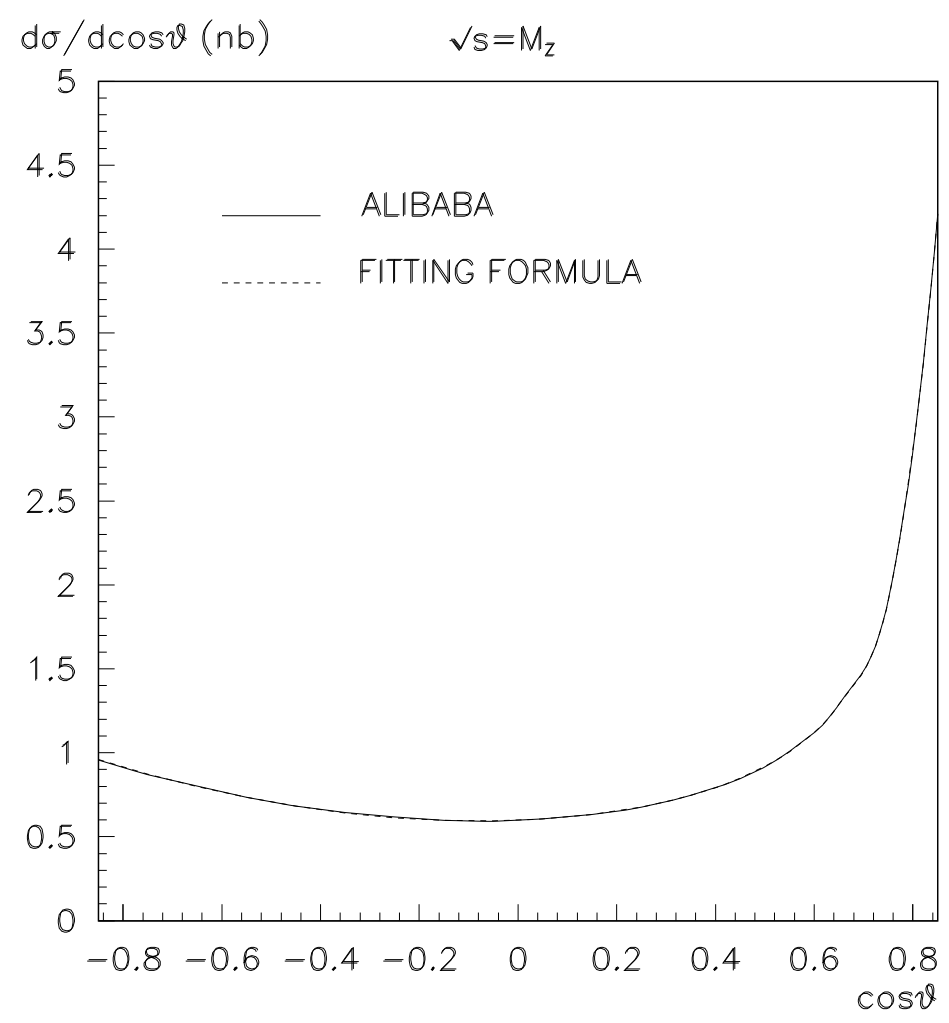

a)

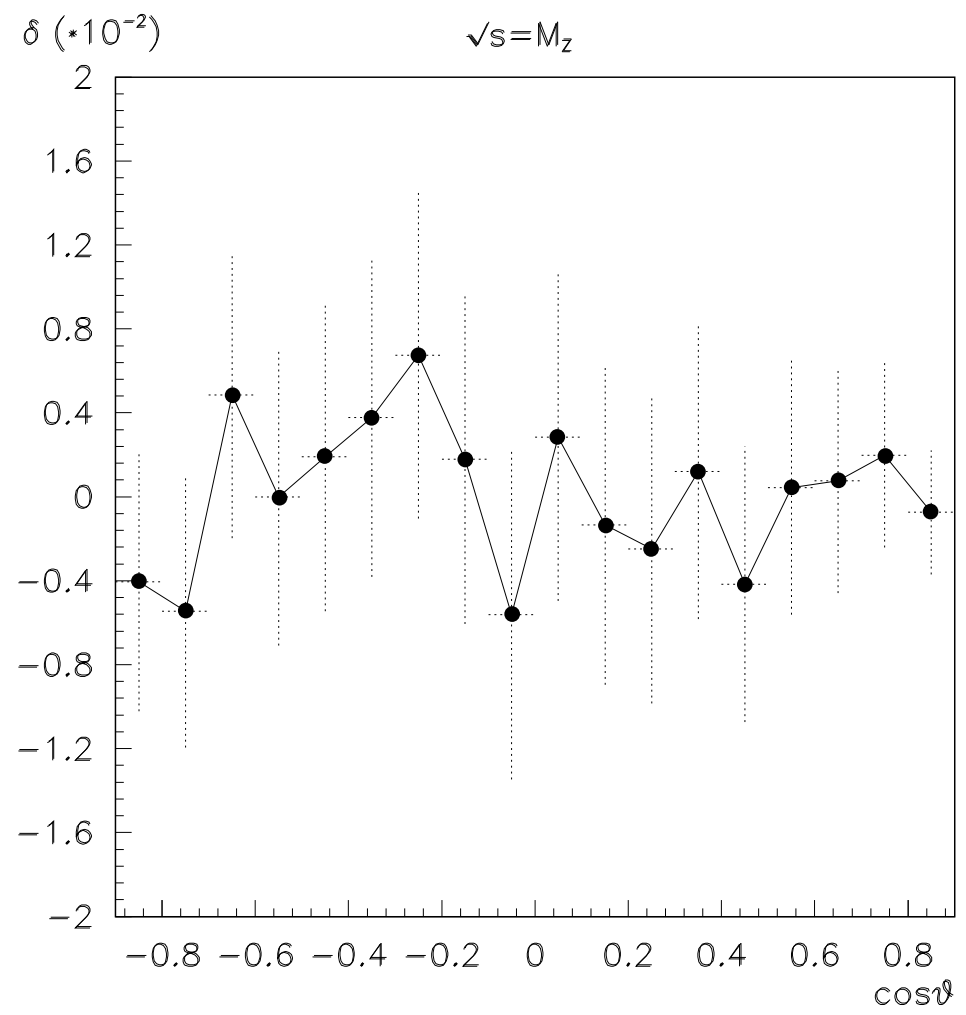

b)

Figure 8: 


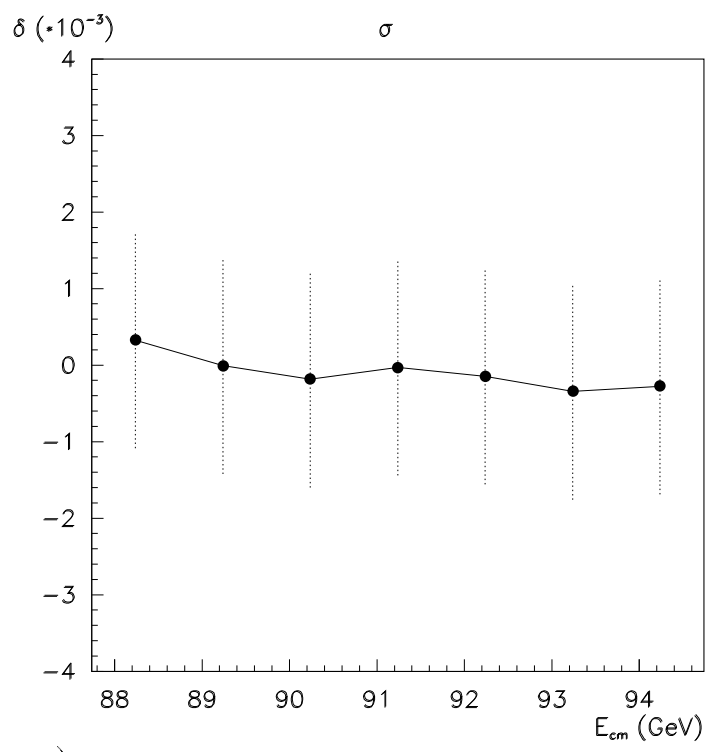

a)

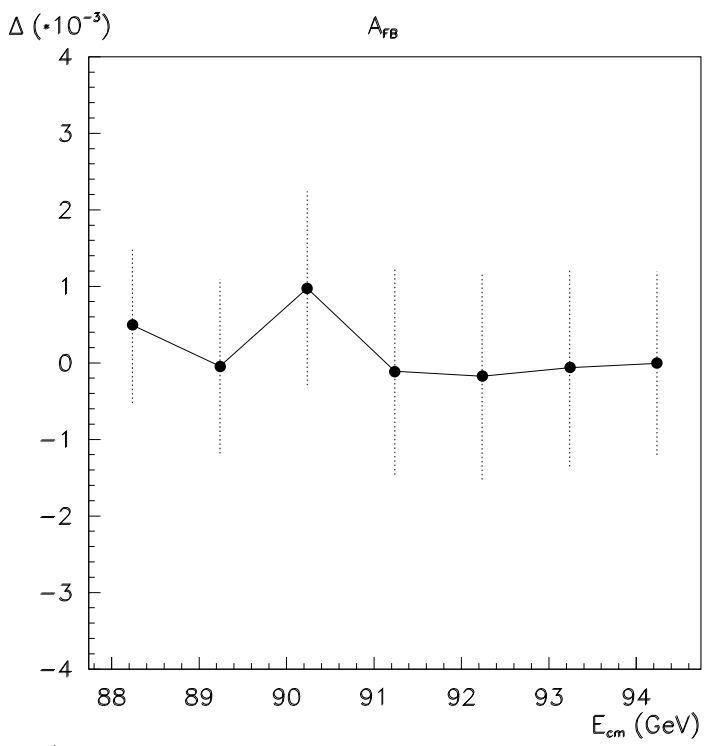

b)

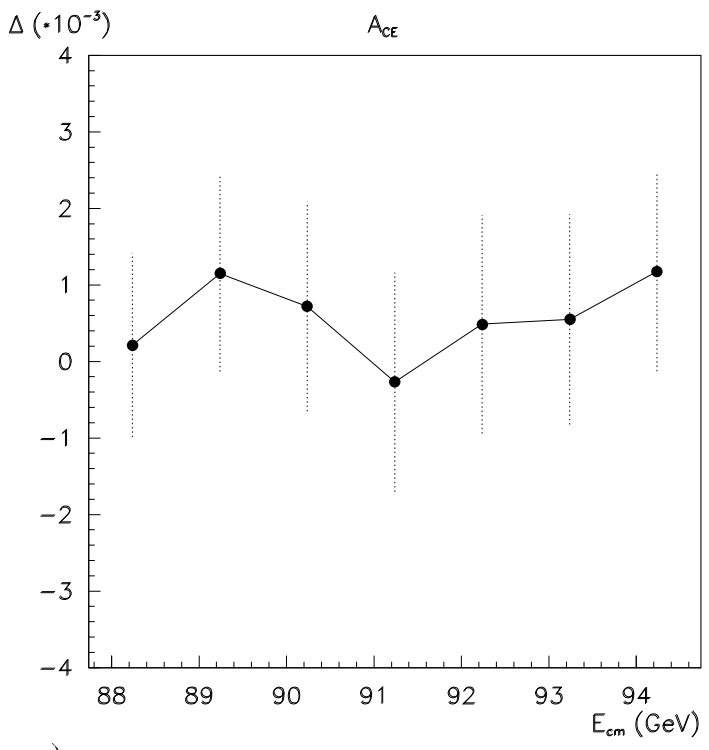

c)

Figure 9: 


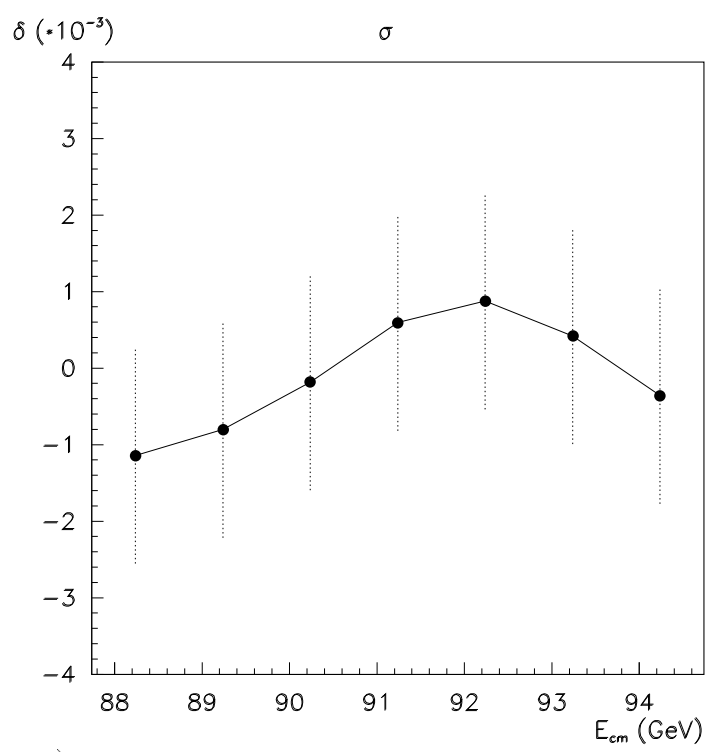

a)

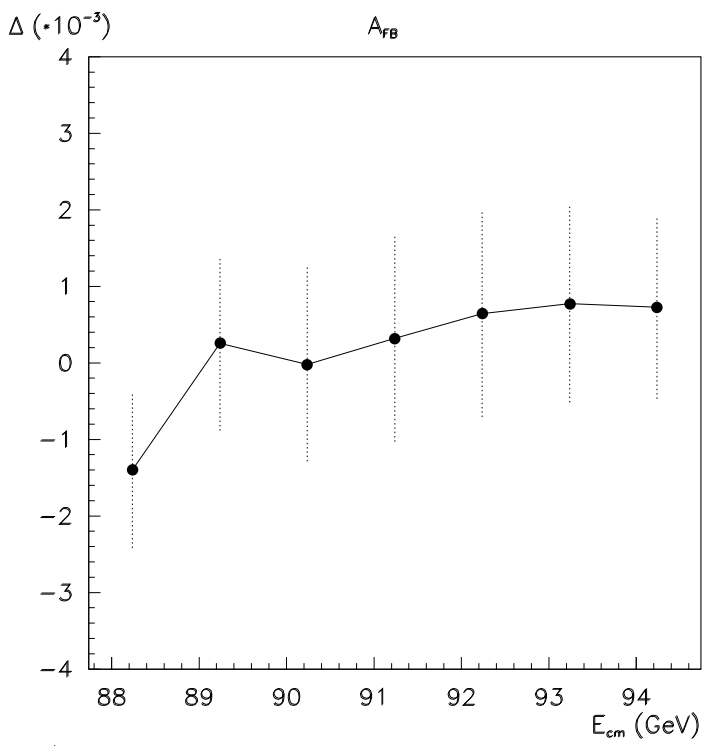

b)

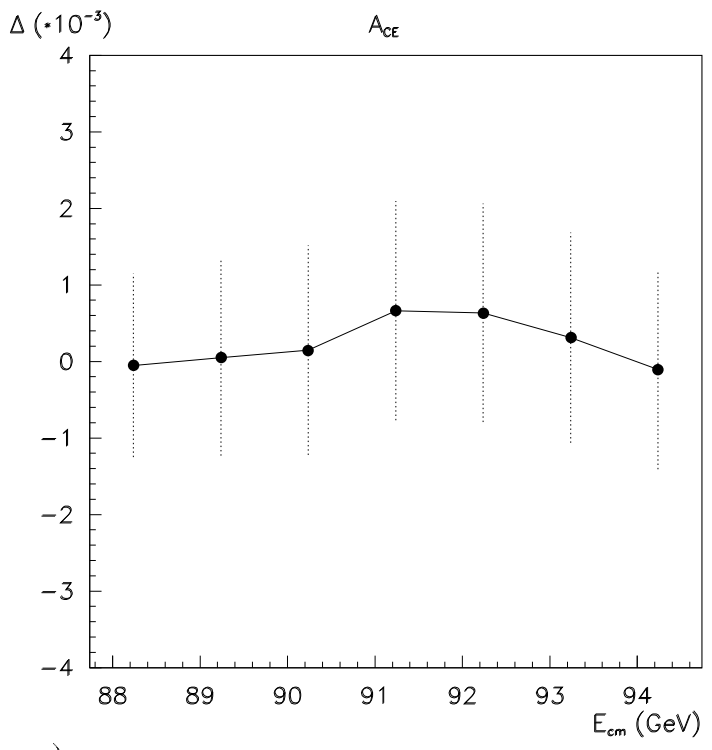

c)

Figure 10: 